\title{
Aging, Jamming, and the Limits of Stability of Amorphous Solids
}

\author{
Vassiliy Lubchenko*,† and Peter G. Wolynes \\ ${ }^{\dagger}$ Departments of Chemistry and Physics, University of Houston, Houston, Texas 77204, United States \\ ${ }^{\ddagger}$ Departments of Chemistry, Physics and Astronomy, and Center for Theoretical Biological Physics, Rice University, Houston, Texas \\ 77005, United States
}

\section{Supporting Information} ABSTRACT: Apart from not having crystallized, supercooled liquids can be
considered as being properly equilibrated and thus can be described by a few thermodynamic control variables. In contrast, glasses and other amorphous solids can be arbitrarily far away from equilibrium and require a description of the history of the conditions under which they formed. In this paper we describe how the locality of interactions intrinsic to finite-dimensional systems affects the stability of amorphous solids far off equilibrium. Our analysis encompasses both structural glasses formed by cooling and colloidal assemblies formed by compression. A diagram outlining regions of marginal stability can be adduced which bears some resemblance to the quasi-equilibrium replica meanfield theory phase diagram of hard sphere glasses in high dimensions but is distinct from that construct in that the diagram describes not true phase transitions but kinetic

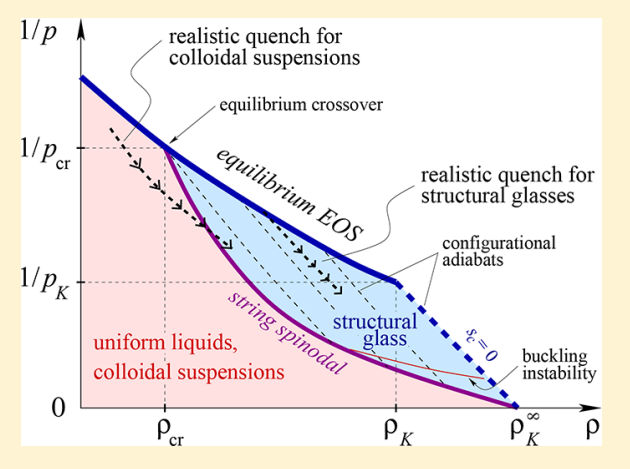
transitions that depend on the preparation protocol. The diagram exhibits two distinct sectors. One sector corresponds to amorphous states with relatively open structures, the other to high density, more closely packed ones. The former transform rapidly owing to there being motions with no free energy barriers; these motions are string-like locally. In the dense region, amorphous systems age via compact activated reconfigurations. The two regimes correspond, in equilibrium, to the collisional or uniform liquid and the so-called landscape regime, respectively. These are separated by a spinodal line of dynamical crossovers. Owing to the rigidity of the surrounding matrix in the landscape, high-density part of the diagram, a sufficiently rapid pressure quench adds compressive energy which also leads to an instability toward string-like motions with near vanishing barriers. Conversely, a dilute collection of rigid particles, such as a colloidal suspension leads, when compressed, to a spatially heterogeneous structure with percolated mechanically stable regions. This jamming corresponds to the onset of activation when the spinodal line is traversed from the low density side. We argue that a stable glass made of sufficiently rigid particles can also be viewed as exhibiting sporadic and localized buckling instabilities that result in local jammed structures. The lines of instability we discuss resemble the Gardner transition of meanfield systems but, in contrast, do not result in true criticality owing to being short-circuited by activated events. The locally marginally stable modes of motion in amorphous solids correspond to secondary relaxation processes in structural glasses. Their relevance to the low temperature anomalies in glasses is also discussed.

\section{Fluctuat nec mergitur}

\section{INTRODUCTION}

Rigidity can emerge, seemingly miraculously, from the interaction of many pieces that are loosely connected: Tents, arches, and domes are among the earliest inventions of humans. Likewise, small fluctuations can sometimes cause such structures to collapse without warning. Although statics has been part of the architectural curriculum since ancient times, ${ }^{1}$ only in the 19th century did Maxwell write down general criteria for building a stable structure out of many unmoving but loosely connected parts. ${ }^{2,3}$ His ideas have resurfaced in the last decades in the description of amorphous solids, such as granular assemblies, at the macroscopic level, and glasses at the molecular size scale. ${ }^{4}$ This modern complex of ideas emphasizes the importance of a "jammed state" of matter, 5,6 which describes an assembly that just barely holds together (i.e., is "marginally stable") but does so under the constraint of a large external pressure.

An apparently distinct view of the emergence of rigidity in amorphous solids more suited to the molecular scale has also emerged, based on the notions of aperiodic crystals and the complexity of the free energy landscapes of disordered systems that have a thermodynamically large number of aperiodic minima. This view comes to terms with the fact that the molecular constituents of amorphous solids are in a state of cease-less atomic motion. The availability of many possible states of repose for static heaps of the constituent particles then not only raises the possibility of flow between these states but also makes such flow inevitable at any finite temperature. Thus,

Special Issue: Benjamin Widom Festschrift

Received: September 26, 2017

Revised: December 7, 2017

Published: December 7, 2017 
Maxwell's criteria for engineering macroscopic structures need to be supplemented, in such molecular systems, by considerations of dynamics and thermodynamics. We see that the rigidity of glasses must be only apparent: Flow simply becomes so slow that an amorphous solid acts as a rigid body on human time scales and the puzzle of rigidity becomes the puzzle of explaining the origin of those extraordinary time scales. The most comprehensive quantitative explanation of these long time scales of motion in amorphous solids is provided by the random first order transition (RFOT) theory of glasses. ${ }^{7,8}$ This theory explains that the slowness arises from the difficulty of finding ways of rearranging loose regions from one jumbled state of repose ("an aperiodic crystal") to another such mechanically stable configuration. Intermittently transiting from one aperiodic crystal arrangement to another, the system can be called a "glassy liquid." This difficulty of finding alternative aperiodic structures grows as the thermal motion diminishes and thereby diminishes the availability of new configurations to which one can move, an impending entropy crisis. If one could reach this entropy crisis, a true phase transition into a unique but aperiodic crystal is envisioned to occur.

The RFOT theory relies on the locality of interactions in order to understand the finite time dynamics but a great deal of progress has been made formalizing RFOT ideas in a meanfield limit appropriate for an infinite dimensional system. Because of the dimensionality, activated flow events become forbidden below a critical temperature and strict "replica symmetry breaking" occurs so that the system can remain forever trapped in one of an exponentially large number of states. This $D=\infty$ meanfield analysis has led also to a rich set of predictions that mirror many of the ideas about jamming that had emerged from the Maxwell-inspired constraint theory of amorphous assemblies. ${ }^{5}$ In the meanfield models, these Maxwell constraints become just marginally satisfied at the so-called Gardner transition, ${ }^{10}$ which corresponds to an isostatic arrangement of particles. While everything is theoretically crisp in the mean field limit, how these ideas are to be carried over to systems in finite dimensions with local interactions, where finite time scales enter, is a question of kinetics not thermodynamics. This topic is addressed in the present article.

Within the RFOT theory, the emergence of stable states of repose out of the equilibrium liquid appears as a type of spinodal. ${ }^{11,12}$ In addition, several lines of argument suggest there is an isomorphism of the emergence of the structural glass state with a spinodal of a random field magnet. ${ }^{13,14}$ At a spinodal, excitations are fractal and resemble strings or percolation clusters. ${ }^{15}$ Using this idea, Stevenson, Schmalian, and Wolynes ${ }^{16}$ described how to locate the point on the equilibrium phase diagram where activated dynamics would start to take over from the collisional transport, the so-called landscape regime. At temperatures above this transition point, mode coupling theory describes the slowing of the dynamics but the MCT singularities are smoothed by the lower barrier activated events which become the dominant relaxation mechanism at still lower temperatures. ${ }^{17-19}$ These fractal excitations also exist in a nonequilibrium glass and there, they give rise to secondary or beta relaxations. ${ }^{20}$ Interestingly Kirkpatrick and Wolynes ${ }^{17}$ speculated that the Gardner transition in the meanfield Potts glasses discussed by Gross et $\mathrm{al}^{21}$ was connected to these secondary relaxations in the structural glasses already in 1987. Lopatin and Ioffe ${ }^{22}$ also discuss the Gardner transition in a meanfield model based on orthogonal density wave tendencies which resemble the models of microemulsions. ${ }^{23}$ The approach we pursue here is to see how these fractal excitations not only remain in the glassy state but can themselves proliferate leading to mechanical instability if an amorphous solid is put under high pressure. This instability occurs because the rigidity of the amorphous matrix allows it to maintain stresses like an arch or dome about a region that reconfigures thereby adding to the driving force for such reconfiguration events. The resulting analysis leads to a diagram that highlights how low barrier processes can arise at high pressure in a finite dimensional system near a jamming point. This diagram bears some resemblance to the well-studied phase diagram of the infinite dimensional hard sphere system but is distinct in several essential aspects. Most importantly, the strict phase transition lines of the $D=\infty$ system turn into dynamical glass transition lines that depend on time scales of observation.

Owing to the presence of discrete spatial and translational symmetries, the analysis of the mechanical stability of periodic crystals can be reduced to computing, for each reciprocal lattice vector, the frequencies of a finite number of vibrational modes that are relatively straightforward to identify. For a broad class of interparticle potentials, such periodic structures can be seen to remain stable just above absolute zero by computing the eigenvalue spectrum of the Hessian of the potential energy landscape. ${ }^{24}$ This analysis can be extended to highly anharmonic systems-like hard spheres at finite temperatures-using the density functional theory (DFT) or selfconsistent phonon theory. ${ }^{25}$ For a pedagogical review, see ref 8 . The hard sphere crystal remains solid at finite temperature even though the particles are virtually never in direct contact except during collisions. Similar stability arguments have been successfully applied to aperiodic collections of both rigid and soft particles. ${ }^{11,26,27}$ The landscapes of those systems develop free energy minima corresponding to localized particles below a crossover centered at a temperature $T_{\mathrm{cr}}$ (or density $\rho_{\mathrm{cr}}$ ). In crucial distinction from perfect periodic crystals, which are nearly unique, the number of aperiodic free energy minima scales exponentially with the system size. The overlap of these many basins of attraction leads to a new route to instability.

The two views of the stability of aperiodic crystals, based on Maxwell-like and landscape-derived notions, respectively, were reconciled in a meanfield model of glassy liquids by Mari, Krzakala, and Kurchan ${ }^{30,31}$ (MKK). A one-stage replica symmetry breaking (RSB) transition emerges at a temperature $T_{A}$, at which the free energy landscape develops many distinct yet equivalent minima. The number of metastable minima scales exponentially with the system size. $\left(T_{\mathrm{A}}\right.$ is the meanfield analog of the temperature $T_{\text {cr. }}{ }^{18}$ ) Because in the meanfield theory the minima are separated by infinite barriers, the system must remain, upon further quenching, in a single one of these minima of the free energy. (If the system is then cooled further, the pressure and density would follow a curve that coincides with the lowest density iso-complexity line in Figure 1. By definition, the complexity is the logarithm of the multiplicity of the free energy minima, per particle.) Yet when Boltzmannaveraged over the totality of the minima, the resulting equation of state is simply a smooth continuation, toward higher densities, of the equilibrium equation of state of the uniform liquid. As a formal construct, one may imagine an ideal nonlocal move set that would allow one to continue to compress the liquid along the so obtained "equilibrium" equation of state and then perform a quench starting at any 


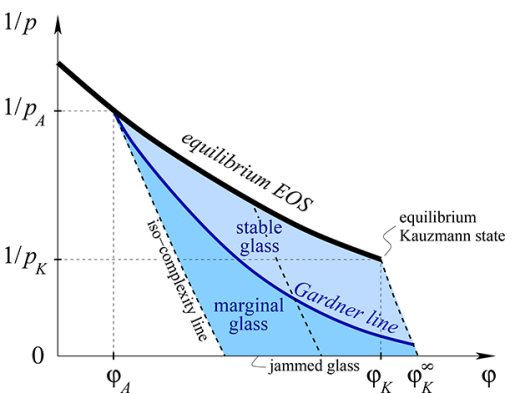

Figure 1. Phase diagram of a meanfield liquid in the $\left(\varphi, p^{-1}\right)$ plane, after ref 28. The quantities $\varphi$ and $p$ are the filling fraction and pressure, respectively. EOS = equation of state. Note reports on the location of the low- $\varphi$ end of the Gardner line vary. ${ }^{29}$

temperature below $T_{\mathrm{A}}$. Such a quench results in the usual glass of laboratory experience and, in the infinite-pressure limit, a jammed configuration that had been arrested in a specific aperiodic minimum, please see the graphical illustration in Figure 1. The behavior of meanfield glassy liquids is however both apparently subtler and richer. On further cooling, the onestage RSB is followed by a full-or continuous - RSB, in which each individual minimum breaks up into an infinite number of sub-basins with a distributed degree of overlap. ${ }^{32}$ This breakup is analogous to the Gardner transitions in $p$-spin models. ${ }^{33}$ Simulations of the MKK model indicate this apparent symmetry breaking is accompanied by a mechanical instability. ${ }^{10}$ Whether this higher order transition will occur in finite dimensions with local interactions is unclear. ${ }^{34}$ Urbani and Biroli $^{35}$ have argued that full RSB is either absent in spatial dimensions less than 6 or perhaps could be conserved through a nonperturbative mechanism. Recent studies of soft polydisperse spheres ${ }^{36}$ suggest that in finite dimensions, quenchinduced instabilities do not span the system but are spatially localized and only sporadic; no susceptibilities seem to diverge.

The appearance of marginally stable modes in the meanfield MKK model and the $D=\infty$ hard sphere glass ${ }^{30,31}$ on approach to jamming ${ }^{5}$ has been proposed ${ }^{28}$ as a possible explanation for anomalies observed in cryogenic glasses, e.g., the Boson Peak and two-level systems. ${ }^{37,38}$ Although suggestive, theories based on this proposal have not yet produced quantitative predictions. In particular, they do not explain the quantitative universality seen in these low temperature properties. ${ }^{39,40}$ On the other hand, arguments ${ }^{41-43}$ within the free-energy landscape framework already quantitatively account for those puzzling cryogenic phenomena by quantizing the reconfigurational motions that normally equilibrate the supercooled liquid. Only a fraction of the molecular motions in question exhibit low enough barriers to be realized near absolute zero quantum mechanically.

Here we treat jamming, aging, and the emergence of rigidity in a unified fashion that goes beyond meanfield theory. The key is accounting for the effects of local density changes during structural relaxations. These density effects are shown to be very significant for deep quenches or highly compressed systems. The results of our analysis are graphically summarized in the diagram in Figure 2. To understand this diagram we must realize that relaxation times can be very long typically but are not strictly infinite in systems with local interactions. Only the transition at the possible entropy crisis where $s_{\mathrm{c}}=0$ can be strictly considered a true phase transition in this diagram. Many states with different pressures and densities throughout the

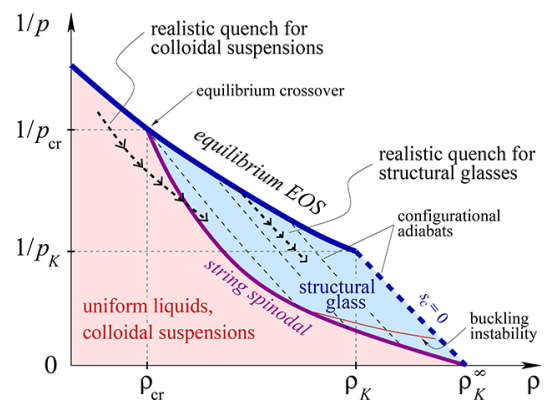

Figure 2. Off-equilibrium diagram of a nonmeanfield liquid in the ( $\rho$, $p^{-1}$ ) plane, where $\rho$ is the number density. $s_{c}$ stands for the configurational entropy. Liquid states cannot exist to the right of the $s_{c}$ $=0$ line, by construction. We note that the configurational adiabats $s_{\mathrm{c}}=$ const are not equivalent to the isocomplexity lines in Figure 1because of finite barriers separating distinct free energy minima-but nevertheless they represent a good approximation to those lines, see text. Note that the "string spinodal" is a crossover, not a sharp boundary, in view of the limited spatial extent of aperiodic free energy minima in finite dimensions. See Figure 4 for approximate evaluation of the sector boundaries for monodisperse hard spheres.

plane can be achieved depending on the sample history. We consider all the states in the colored part of the diagram as being potentially accessible. Even under uniform cooling or compression, many different quenching protocols can be realized depending on the speed of quenching and the precise sequence of cooling and compression or heating and pressure release.

The system falls out of equilibrium when the relaxation and quenching time scales match. Quenches that start below the crossover to activated transport result in a frozen glass (the blue region on the diagram) whose structure initially is not significantly configurationally perturbed relative to the original equilibrated liquid. Each particle largely maintains the same set of neighbors which had formed a transient cage around it already in the equilibrium state. Still, aging toward lower free energy states will occur via local, compact activated processes, generally accompanied by a significant increase in the density along with a decrease in the pressure; these aging events can be quantified as we have described in our previous work on aging. ${ }^{44}$ Because in finite dimensions, individual free energy minima are not infinitely long-lived and are defined over finite regions in space, the configurational entropy is not equivalent to the complexity, which strictly speaking requires infinite barriers for unambiguous definition, a possibility in mean field. Nevertheless, the two concepts become practicallly interchangeable when the barriers separating distinct free energy minima are sufficiently high. At the particle level, this corresponds with there being a sufficiently large time-scale separation between diffusional and vibrational degrees of freedom.

Following an activated aging event, the existing cages in the aperiodic crystal are locally replaced by slightly different cages. In the reconfiguring region, individual particles move distances comparable to the typical vibrational displacement within a cage. Each particle nevertheless remains surrounded by the same set of nearest neighbors as before the event. Several reconfiguration events would be needed for the identity of the neighbors to be fully reset. Save for effects of volume relaxation, which we will now focus on, the above microscopic picture is very much like that developed earlier by us where we focused primarily of shallow thermal quenches. ${ }^{44} \mathrm{We}$ see that 
amorphous solids can be regarded as mechanically stable at all length scales below a cooperativity size $N^{*}$; this scale depends on the time scale of the quench. Amorphous solids are only metastable on scales larger than $N^{*}$, which is determined by an argument that resembles nucleation theory.

Suppose we continue a thermal quench. Once the quench becomes sufficiently deep, the vibrational amplitude of a given particle may become less than the gap to some of its neighbors so the particle finds itself colliding with a smaller set of neighbors than it was making contact with in the beginning of the quench. This breaking of local symmetry comes from the individual cages not being strictly isotropic when the system comes out of equilibrium. If the number of long-lived collisional contacts becomes less than the number of Maxwell constraints, the system becomes locally unstable. The microscopic consequences of the resulting instability strongly depend both on the density and pressure. We emphasize that for systems away from equilibrium, there is no one-to-one correspondence between pressure and density (at a fixed temperature). Both parameters must be specified separately in order to describe both instability and jamming at high pressures.

We will see that for a sufficiently deep quench, the local relaxations result in such a significant increase in coordination, and a concomitant local pressure decrease, that the energy released in reconfiguring can drive the barrier for reconfiguration to zero. Particle in the reconfigured region will move a distance significantly exceeding the typical vibrational amplitude thus destroying the existing cages and then forming new cages using a distinct set of neighbors, in an avalanche-like fashion. When one particle escapes its existing cage, the avalanche motion will resemble a string whose direction changes. The avalanche also can branch leading to a fractal percolation-like cluster of rearranged particles. This instability toward fractal motions is well documented in equilibrated glassy liquids: ${ }^{45}$ The mathematics resembles a "stringdeconfinement" transition and has been described by Stevenson, Schmalian, Wolynes. ${ }^{16}$ When strings proliferate, the motions change from being largely activated to collisional ${ }^{18}$ and can be described by extended mode-coupling theory. This transition is a crossover spinodal. We will also observe that for sufficiently rigid particles, the string instability seems to be related to a set of significantly milder instabilities in which particles confined to anisotropic cages will have to choose one end of the cage over the other. This other end of the cage will partially collapse, or "buckle", upon further quenching, resulting in a local jamming event.

We note that achieving sufficiently high dimensionless pressures in molecular systems, in order to encounter the transitions to marginal stability, is hard. For these reasons, we believe quench-induced marginal stability is unlikely to underlie the low temperature anomalies observed in the laboratory for structural glasses. On the other hand, in granular and colloidal particles, these high dimensionless pressures can be achieved but these systems are made of larger constituents and are difficult to equilibrate at high densities owing to their intrinsically long time scales for particle motion. Colloidal assemblies are typically prepared at pressures and densities where strings nucleate largely unimpeded. Once frozen, such systems will age largely by string-like motions, not compact reconfigurations. We shall see that when such systems are densified past the spinodal, the local increases in density lead rapidly to activation barriers so the aging will be significantly slowed down. As a result, colloidal assemblies will become spatially heterogeneous collections of nearly mechanically unstable configurations. Being trapped in such configurations on experimentally relevant time scales will lead to a nearly marginally stable, jammed structure at infinite pressure. A quench starting from the low density phase and crossing the string spinodal will rapidly lead to the onset of apparent mechanical rigidity.

True criticality is not expected at the string spinodal or the buckling instability in this high pressure region because at finite temperature, long-range correlations will be destroyed by the activated reconfigurations themselves just as at equilibrium, the mode-coupling singularity is short-cut by activated reconfigurations. ${ }^{17}$ The lack of such criticality excludes the possibility of a true macroscopic instability, hence the article's epigraph. Nevertheless, long-range correlations may well be observed on times less than the activated reconfiguration time.

\section{RECONFIGURATION BARRIERS AND THE MARGINAL STABILITY IN FAR-FROM-EQUILIBRIUM GLASSES}

It is useful to set the stage for the detailed analysis by reviewing some basic notions about glassy behavior. The equilibrium liquid equation of state, which is graphically shown in Figure 3

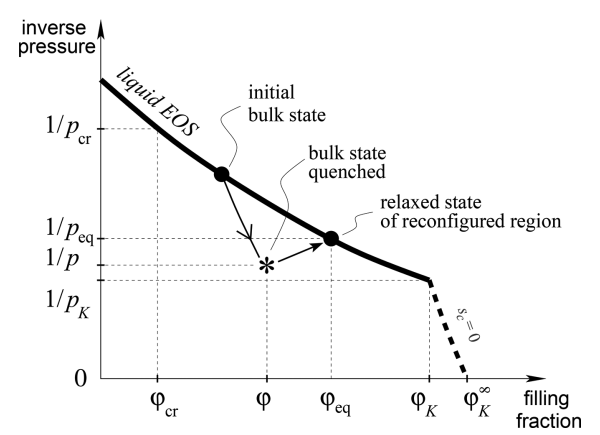

Figure 3. Illustration of the liquid equation of state (EOS), a quenching protocol to a state $(\varphi, p)$, and the resulting state $\left(\varphi_{\text {eq }}, p_{\text {eq }}\right)$ of a compact reconfigured region.

by the thick line, ignores the possibility of crystallization to a conventional periodic structure. (The ultimate fate of a supercooled liquid is to crystallize. ${ }^{46}$ ) At a filling fraction $\varphi_{\mathrm{cr}}$, the liquid nevertheless breaks translational symmetry aperiodically. At a sufficient density, the free energy computed say by density functional theory is no longer minimized by a spatially uniform density profile. ${ }^{11}$ Instead, a thermodynamically optimal density profile can be well approximated by a superposition of narrow Gaussian peaks centered at sites $\boldsymbol{r}_{i}$ of an aperiodic lattice: ${ }^{11,47}$

$$
\rho(\boldsymbol{r})=(\alpha / \pi)^{3 / 2} \sum_{i} e^{-\alpha\left(\boldsymbol{r}-\boldsymbol{r}_{i}\right)^{2}}
$$

The quantity $\alpha$ determines the magnitude of the thermal fluctuations of particles localized at $\left\{\boldsymbol{r}_{i}\right\}$. The lattice specified by $\left\{\boldsymbol{r}_{i}\right\}$ is associated with a mechanically stable structure; the eigenvalues of the Hessian of the free energy functional should be non-negative. Consequently, transitions to an alternative aperiodic lattice must occur by activation. In the absence of fluctuations in the magnitude of the order parameter $\alpha$, the onset of mechanical metastability with this ansatz occurs at a sharply defined temperature $T_{\mathrm{A}}$. In finite dimensions, the local order parameter can vary from site to site. This allows 


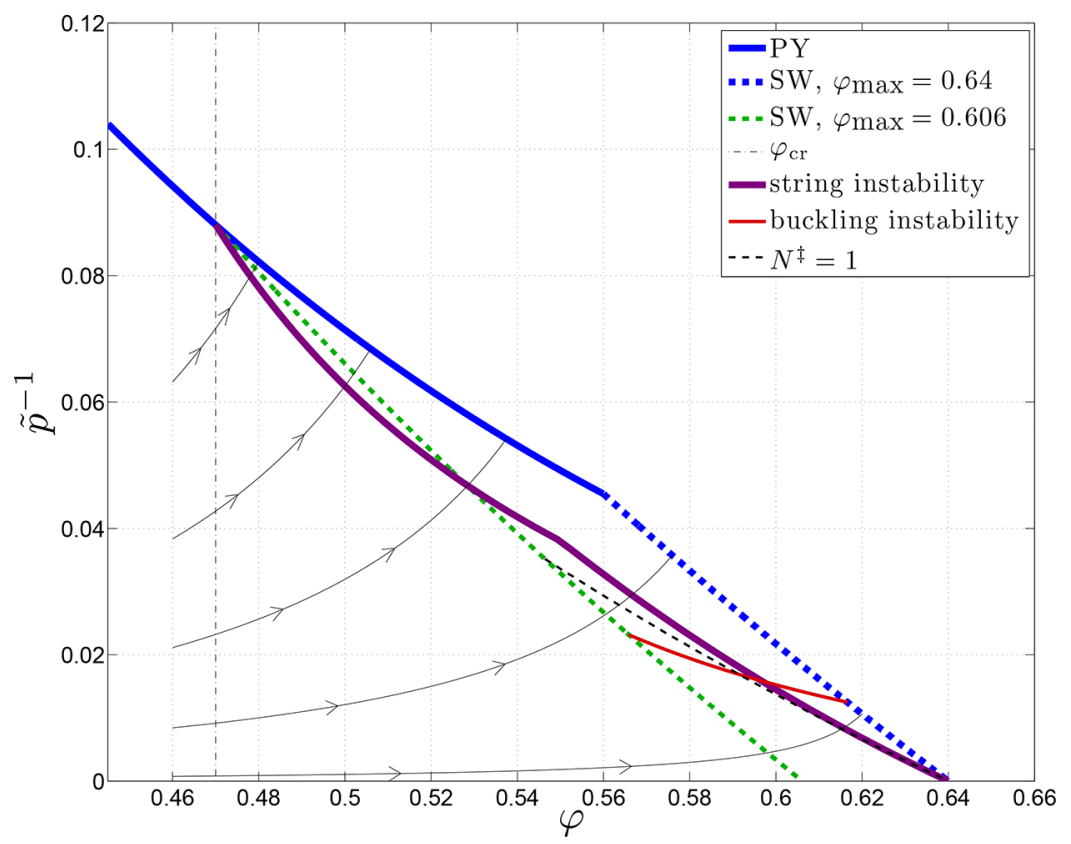

Figure 4. Off-equilibrium diagram for monodisperse hard spheres. The equilibrium equation of state (EOS) is approximated using the PercusYevick $^{59}$ (PY) form. The Salsburg-Wood (SW) form from eq 8 is used to approximate the configurational adiabats that cross the equilibrium EOS near the equilibrium crossover and the putative Kauzmann state.

excitations that lead to a significant lowering of the onset temperature of rigidity from the meanfield density functional value: $T_{\mathrm{cr}}<T_{\mathrm{A}}\left(\rho_{\mathrm{cr}}>\rho_{\mathrm{A}}\right) \cdot{ }^{18,27}$ Owing to these excitations, the onset also becomes a gradual crossover. ${ }^{8,13,48}$ The density $\rho_{\mathrm{cr}}$ is the value of density at which the crossover to activated dynamics is centered.

No thermodynamic quantities experience a singularity at $\rho_{\mathrm{A}}$ in the meanfield theory nor at $\rho_{\mathrm{cr}}$ in the finite-dimensional analysis. This has been made clear by using the replica symmetry breaking formalism. ${ }^{49}$ At temperatures below $T_{\mathrm{cr}}$ the aperiodic structure represented by $\left\{\boldsymbol{r}_{i}\right\}$ persists locally for long times compared to vibrational times but eventually transforms in an activated fashion so that the liquid can flow. The corresponding degrees of freedom labeling the distinct mechanically stable configurations are usually referred to as "configurational" degrees of freedom. Their thermodynamics is largely decoupled from the vibrations, see ref 8 . The key thermodynamic quantity is the configurational part of the entropy, which reflects the multiplicity of the aperiodic free energy minima.

To provide a concrete but, at best, semiquantitative illustration, we will use assemblies of monodisperse spheres as the model liquid. Focusing on this system allows us also to make connection with existing meanfield studies of hard spheres. It also allows us to find explicit numerical estimates using approximate equations of state of venerable vintage. For hard spheres, it is convenient to work with the filling fraction $\varphi$ :

$$
\varphi \equiv \rho\left(\pi \sigma_{\mathrm{hs}}^{3} / 6\right)
$$

where $\sigma_{\mathrm{hs}}$ is the diameter of the sphere. Henceforth, we will use the number density $\rho$ and the filling fraction $\varphi$ interchangeably. Real molecules are not hard spheres. Their interactions are harshly repulsive but ultimately soft. At finite temperature, we can consider molecules to have an effective core diameter ${ }^{50}$ that can be determined using an unambiguous prescription such as those due to Barker and Henderson ${ }^{51}$ or due to Weeks,
Chandler, and Anderson ${ }^{52}$ or their appropriate generalizations for high density. ${ }^{53}$ A key quantity in our analysis will be the bulk modulus. The expression for this quantity looks the same whether written in terms of $\rho$ or $\varphi$ :

$$
K \equiv-V \frac{\partial p}{\partial V}=\frac{\partial p}{\partial \ln \rho}=\frac{\partial p}{\partial \ln \varphi}
$$

After orienting ourselves by having looked at the quasiequilibrium curve, we now turn to discuss the glasses that result from quenches that start at densities above $\varphi_{\mathrm{cr}}$. In this regime, suppose a quench is made that allows for vibrational equilibration but is so fast that no configurational equilibration whatsoever can occur. Each particle would thus maintain the same set of neighbors as it had in the beginning of the quench. In a meanfield sense, this neighbor set would be described by a coordination number $z$. $z$ ultimately will determine the maximum putative density that can be achieved by the infinitely rapid quench. The equation of state of a hard sphere system, when confined to a single mechanically stable basin, is then, approximately, $p \simeq k_{\mathrm{B}} T / d a^{2},{ }^{27}$ where the quantity $d$ stands for the typical particle-particle gap in this basin and depends on the coordination. The quantity $a$, defined as

$$
a \equiv 1 / \rho^{1 / 3}
$$

is the volumetric size of a particle. The corresponding bulk modulus in a quenched frozen state, $K \equiv-V(\partial p / \partial V)_{\mathrm{T}} \simeq k_{\mathrm{B}} T /$ $3 d^{2} a$, thus exhibits a simple, ideal-gas like scaling relation with the pressure:

$$
\tilde{K} \simeq A \tilde{p}^{2}, \text { if } \tilde{p} \rightarrow \infty
$$

In our analysis, it is convenient to use the dimensionless forms of the two quantities:

$$
\begin{gathered}
\tilde{K} \equiv K / \rho k_{\mathrm{B}} T \\
\tilde{p} \equiv p / \rho k_{\mathrm{B}} T
\end{gathered}
$$


The coefficient $A$ reflects the space dimensionality; in three dimensions, $A=1 / 3$. The relation is satisfied by the SalsburgWood (SW) functional form: ${ }^{53,54}$

$$
\tilde{p}=\frac{3}{\varphi_{\max } / \varphi-1}+1
$$

where $\varphi_{\max }$ is a maximal putative density achievable by rapidly quenching the starting structure; $\varphi_{\max }$ depends on the coordination in the original frozen state. It is a function of the density and temperature where the system first fell out of equilibrium. ${ }^{55-57}$ The magnitude of $\varphi_{\max }$ reflects the coordination number $z$, which is used in theories based on counting Maxwell constraints. Now, since reconfigurational motions are not allowed when making an infinitely rapid quench, the corresponding configurational heat capacity is zero, so the configurational entropy remains constant during the quench: $s_{c}$ = const. The above protocol is approximately followed during routine thermal quenches in the laboratory even though some aging is expected to occur, if one waits long enough after first falling out of equilibrium. We discussed the inhomogeneous structure that occurs after waiting in our earlier paper. ${ }^{44}$ Now, there is a putative density, $\varphi_{\mathrm{K}}$, above which $s_{\mathrm{c}}=0$, in equilibrium. The third law makes this a fixed lower bound on the glass transition temperature, ${ }^{9}$ which can only be reached for infinitely slow cooling. This ideal, "Kauzmann" state corresponds with the most stable aperiodic crystal and exists for liquids in $D=\infty .{ }^{58}$ We show the $s_{c}=0$ line in Figure 3 as the putative limiting case for the slowest possible quench. At any rate, the vibrational part of the compressibility for particles depends on the particle-particle gaps but does not depend very much on the precise coordination pattern. The slopes of the $s_{c}$ $=$ const lines, shown by thin dashed lines on Figure 2, are thus numerically similar for different values of $s_{c}$. The region putatively accessible by such "conventional" quenches is depicted in blue in the latter Figure. Configurational adiabats for monodisperse hard spheres, as approximated using the Salsburg-Wood form, are shown in Figure 4.

We now analyze the stability of the amorphous solid and the spatial extent and kinetics of structural relaxation of the amorphous states that were obtained by quenching an equilibrated liquid with $\varphi>\varphi_{\mathrm{cr}}$. Because a rapidly quenched state is off-equilibrium, any further relaxation technically corresponds with aging. Conversely, if we are still to be confined to a single basin, quenches must be extraordinarily fast. Still, this means that the Salsburg-Wood adiabats for relatively low values of $\varphi_{\max }$ can be formally defined and accessed.

Here we present a quantitative framework to describe aging that generalizes the one that was developed earlier by us ${ }^{44}$ and extended by Wolynes and co-workers to include for the possibility of achieving states of marginal stability. ${ }^{16,60,61} \mathrm{We}$ first review those results that assumed that individual, aginginduced displacements do not significantly exceed the typical vibrational amplitude. We do this first for reconfigurations that are relatively compact and then generalize to the stringy or fractal case. The free energy cost for a compact region to rearrange, in a fixed environment, is well approximated by a sum of a bulk contribution $\Delta g N$ and a mismatch penalty $\gamma N^{1 / 2}:^{13,44,48}$

$$
F(N)=\Delta g N+\gamma N^{1 / 2}
$$

where $N$ is the number of reconfigured particles. The squareroot scaling of the mismatch penalty is slower, at large $\mathrm{N}$, than the conventional surface scaling $N^{2 / 3}$. This nonclassical scaling of the mismatch between two aperiodic free energy minima comes about because the strained region at the border between the displaced particles and the original environment can typically lower its energy by as much as $\sim N^{1 / 2}$ by locally replacing any region with an equivalent structure chosen out of the random ensemble. ${ }^{13,48,62,63}$ This effects parallels the wetting effect discovered by Villain for the random field Ising magnet. ${ }^{64}$ This free energy lowering naturally scales with the magnitude of Gibbs free energy fluctuations ${ }^{13,62}$ and is thus approximately determined by the bulk modulus: ${ }^{8,62}$

$$
\tilde{\gamma} \equiv \gamma / k_{\mathrm{B}} T=\tilde{K}^{1 / 2}
$$

There are other, numerically smaller contributions to the mismatch penalty, ${ }^{62}$ which we neglect here. In any event, the mismatch penalty between two random structures-the originally quenched structure and the structure it can locally relax to-must be bounded from above by the bulk modulus of the stiffer of the two structures. A better approximation is obtained by noting that all lattice properties depend continuously on the coordinates ${ }^{63}$ and so from here on, we will assume the mismatch penalty is determined by the value of the bulk modulus at the interface. We believe that eq 9 is valid in all dimensions above the lower critical dimension. ${ }^{13}$ We also note, nevertheless, that the corresponding interface cost is expected to diverge in the meanfield limit of long-range forces. $^{65}$

Consider now a quench toward the state denoted by the asterisk on Figure 3. The external pressure $p$ is higher than what it would be in equilibrium, at the density and temperature in question, because the structure we start with is more open than it would be in macroscopic equilibrium. The reconfiguring region will thus relax to a state having a lower specific volume-in which coordination is higher-and equilibrate to some new value of pressure, call it $p_{\text {eq }}$, which is less than the external pressure, $p_{\mathrm{eq}}<p$. The mechanically equilibrated pressure in the reconfigured region and its immediate surrounding will be lower than the external pressure because the environment is rigid! The internal pressure can only partially accommodate for the contraction of the reconfigured region since the shear modulus $\mu$ of the environment is finite. These notions are graphically illustrated in Figure 5.

The residual pressure mismatch is only modest for shallow quenches. ${ }^{8}$ We therefore neglected this mismatch in our earlier analysis of aging. ${ }^{44}$ Here, in contrast, we want to estimate the relaxation of pressure inside the reconfigured region for

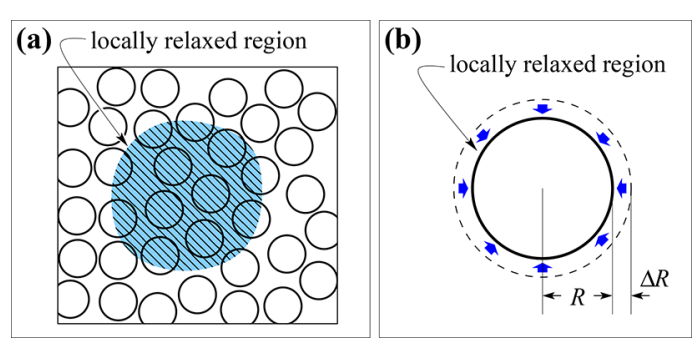

Figure 5. Cartoon of an aged region, in which the pressure and specific volume have decreased as a result of a reconfiguration event. Note the coordination in the rearranged central region will be larger than that in the bulk. 
arbitrarily high values of the external pressure $p$; such high pressures are needed to achieve jamming. To calculate the extent of density relaxation, first suppose the volume of the relaxed region will change by $\Delta V$ following an aging event. The relation between the relative volume change, or packing fraction change, $\Delta V / V=-\Delta \varphi / \varphi$ and the pressure drop can be estimated using continuum, linear elasticity theory. This has been described by Landau and Lifshits in the solved Problem 7.2 from ref 66, see also the Appendix of ref 8 . We briefly reprise the argument here. For a spherically symmetric geometry, the deformation $u$ outside the reconfigured region is directed along the radius-vector while its magnitude is given by ${ }^{66} u(r)=a r+b / r^{2}$. The linear term, $a r$, describes the spatially uniform deformation caused by the externally applied hydrostatic pressure, while the second term, $b / r^{2}$, takes care of spatial inhomogeneities near the inclusion, if any. Note that the deformation in the region's environment is pure shear even though the total volume of the sample will decrease as a result of the reconfiguration event by a fraction of $\Delta V{ }^{8}$ The resulting radial stress is given by the expression ${ }^{66} \sigma_{r r}(r)=3 \mathrm{Ka}-4 \mu \mathrm{b} / \mathrm{r}^{3}$. The coefficients $a$ and $b$ are fixed by the boundary conditions at the interface of the inclusion $\sigma_{r r}(R)=-p_{\mathrm{eq}}$ and spatial infinity $\sigma_{r r}(\infty)=-p$, yielding $b=-\left(p-p_{\text {eq }}\right) R^{3} / 4 \mu$. Thus, the displacement of the boundary of the reconfigured region, relative to its position before reconfiguration in the presence of external pressure $p$, is given by $\Delta R=u(R)-a R=-R(p-$ $\left.p_{\text {eq }}\right) / 4 \mu$. In terms of the relative volume change $-\Delta \varphi / \varphi=$ $\Delta V / V=3 \Delta R / R$ of the reconfigured region, this yields for the pressure mismatch between the equilibrated region and the bulk of the sample:

$$
p-p_{\text {eq }}=\frac{4}{3} \frac{\Delta \varphi}{\varphi} \mu_{\mathrm{eq}}
$$

Since the equation is correct up to terms of order $\Delta \varphi^{2}, \varphi$ on the r.h.s. can stand for the density of either the initial or final state; we will set $\varphi=\varphi_{\text {eq }}$ for convenience. According to eq 5, the elasticity is clearly nonlinear in the high pressure limit. Still, eq 11 indicates that most of the deformation will take place in the softer regions, where the elastic constants are smallest: $\Delta \varphi$ $\propto 1 / \mu$. Accordingly, we use in eq 11 the value of the shear modulus pertaining to pressure $p_{\mathrm{eq}}$ as pertinent to the region just outside the reconfigured region. The label "eq" at $\mu_{\mathrm{eq}}$ in the r.h.s. explicitly refers to the fact. In any event, eq 11 becomes more accurate the smaller $\Delta \varphi$ is. Now, the value of pressure in the relaxed region is determined by simultaneous solution of eq 11 and the equilibrium equation of state from Figure 3. At lower pressures (higher temperatures) one follows the $s_{\mathrm{c}}>0$ branch, but at higher pressures (lower temperatures) one follows the "Kauzmann" portion $s_{\mathrm{c}}=0$ :

$$
p_{\mathrm{eq}}=p_{\mathrm{eEOS}}\left(\varphi_{\mathrm{eq}}\right)
$$

where the label "eEOS" signifies "equilibrium equation of state". It is not difficult to convince oneself that such a solution to the nonlinear elastic balance always exists, as is explicitly illustrated in Figure 4 for hard spheres: As a practical matter, it is most convenient to first find and plot the sets of quenched states $(\varphi$, $p$ ) that would relax locally into a particular equilibrated state $\left(\varphi_{\text {eq }}, p_{\text {eq }}\right)$, because the equilibrium EOS has a discontinuity in slope at the Kauzmann state $\left(\varphi_{\mathrm{K}}, p_{\mathrm{K}}\right)$. Examples of such paths of relaxation are shown in Figure 4 as curves with arrows. By the collapse of the interior of a reconfigured region, any initial state belonging to such a curve would relax, within that region, to the equilibrium state located at the high density end of the curve. To avoid confusion we remind the reader that the intermediate portions of these "local-collapse curves" do not correspond to kinetic intermediates; a single aging event leads the system directly to the state on the equilibrium equation-ofstate line in the interior of the aged region. To solve for pairs $(\varphi, p),\left(\varphi_{\mathrm{eq}}, p_{\mathrm{eq}}\right)$ of quenched and relaxed states, respectively, we have expressed the shear modulus through the bulk modulus and the Poisson ratio $\sigma=(3 K-2 \mu) / 2(3 K+\mu)$ :

$$
\mu=\frac{3(1-2 \sigma)}{2(1+\sigma)} K_{s_{\mathrm{c}}}
$$

For concreteness, we have also assumed a value of the Poisson ratio characteristic of the aperiodic crystal made of hard spheres, ${ }^{26}$ see the Supporting Information for details. It is essential that the bulk modulus used in eqs 11 and 13 be not the isothermal kind $(\partial p / \partial \ln \varphi)_{T}$, but the one computed along the appropriate $s_{c}=$ const lines, as is emphasized by the subscript $s_{c}$ in eq 13 . This is, again, because the surrounding matrix is assumed to relax exclusively by vibrational readjustments.

Because the overall sample is maintained at constant external pressure and temperature, the relevant driving force in eq 9 is the Gibbs free energy. ${ }^{67}$ This can be seen by writing down the expression for the probability for a system to have energy $E$ and volume $V$ when subject to an external pressure $p$ and temperature $T: p(E, V) \propto e^{-\beta(E-T S+p V)}$. The total entropy includes both a vibrational and configurational contribution. Since aging-induced escape occurs from a particular aperiodic minimum, the configurational entropy of the initial state is zero by construction. Thus, in the usual way, ${ }^{44,62}$ the driving force for reconfiguration is given by the change in the Gibbs free energy of an individual aperiodic state and an entropic contribution due to the multiplicity of the target state. Per particle, it is given by the quantity

$$
\Delta g=p_{\mathrm{eq}} / \rho_{\mathrm{eq}}-p / \rho-T s_{\mathrm{c}}\left(p_{\mathrm{eq}}\right)-T \Delta s_{\mathrm{vibr}}<0
$$

The first two terms on the r.h.s. account for the enthalpic stabilization of the region due to the pressure drop. This is nonzero because the environment is a rigid solid, not a stressfree fluid, as already discussed. The $-T s_{\mathrm{c}}\left(p_{\mathrm{eq}}\right)$ terms accounts for the multiplicity of the free energy minima in the equilibrium state, and the term $-T \Delta s_{\text {vibr }}$ corresponds with the stabilization due to the increase in the vibrational entropy upon reconfiguration. The $\Delta s_{\text {vibr }}$ term stems from the increased spacing $d$ between the particles in a state with a higher coordination number. Since for rigid objects $d \propto 1 / p$, one can approximately write

$$
\Delta s_{\text {vibr }}=3 \ln \left(p / p_{\text {eq }}\right)
$$

For soft particles, the dependence of the vibrational amplitude, and thus vibrational entropy, on pressure is more complicated and, furthermore, system-dependent. In any event, this dependence is significantly weaker than the already mild, logarithmic dependence in eq 15. There is another potential contribution to the driving force in eq 14, viz., any vibrational energy difference $\Delta E_{\text {vibr }}$ between the quenched and target state of the reconfigured region, which would generally depend on anharmonicity and quantum effects. For rigid objects, this difference is strictly zero. For classical oscillators, it also vanishes in the harmonic limit. Accounting for effects of anharmonicity would not significantly improve the accuracy of 

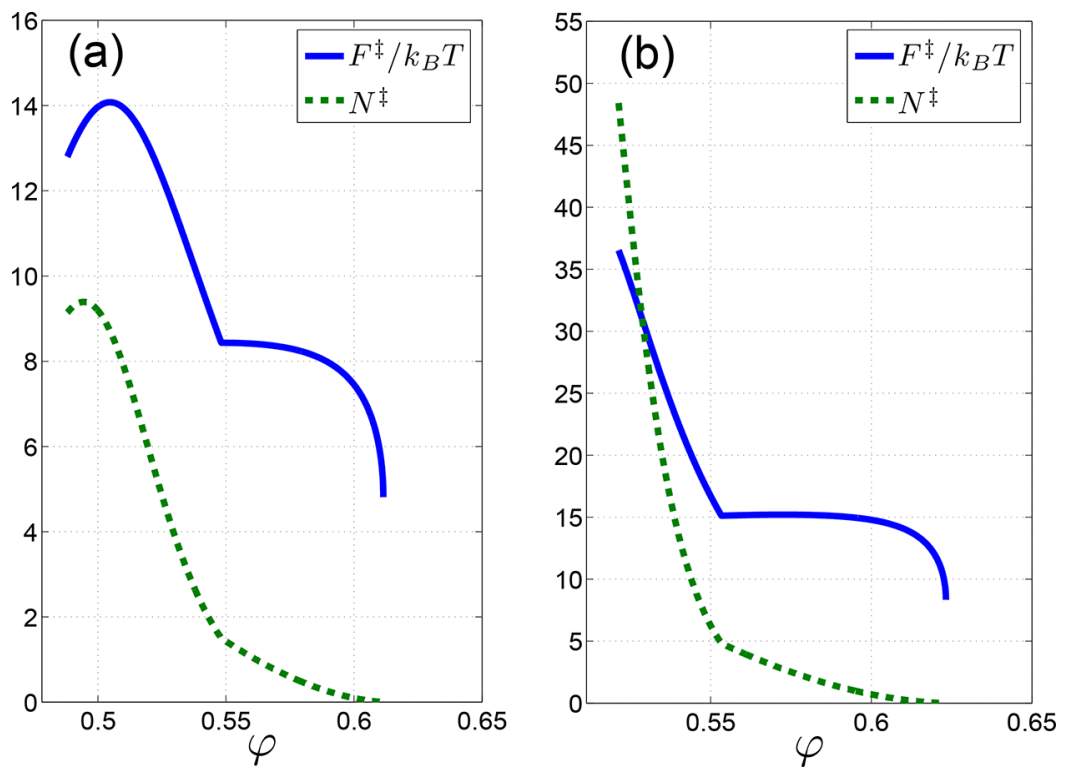

Figure 6. Density dependence of the dimensionless activation barrier and critical size for structures obtained by quenching along Salsburg-Wood (SW) adiabats that intersect the equilibrium $\operatorname{EOS}$ at $\varphi=0.4880\left(\varphi_{\max }=0.6114\right)$ in panel (a), and $\varphi=0.5213\left(\varphi_{\max }=0.6232\right)$ in panel $(\mathrm{b})$.

approximation, see the discussion of eq 11 ; these effects, if any, will be therefore ignored. Finally, we inquire whether there is an enthalpic stabilization of the environment of the reconfigured region. For linear elasticity, the environment experiences only shear but no uniform dilation, as already mentioned. Thus, the radial stress is exactly compensated by the lateral stress and so, no stabilization is expected at this level of approximation. The next order term will scale quadratically with $\Delta \varphi$. This contribution could represent a substantial correction to the driving force for nucleation within a rigid medium (as has been argued for crystallization ${ }^{68}$ ), but including it would exceed the needed accuracy for the jamming question; this contribution will be ignored henceforth. To avoid confusion we note that the free energy cost of creating an interface between the environment and the reconfigured region has been already included through the mismatch penalty $\gamma \mathrm{N}^{1 / 2}$.

Now, the critical size $N^{\ddagger}$ at which the free energy profile (9) for compact reconfiguration events reaches its saddle-point value is easily computed:

$$
N^{\ddagger}=\left(\frac{\gamma}{2 \Delta g}\right)^{2}
$$

while the activation barrier itself equals:

$$
F_{\alpha}^{\ddagger}=\gamma^{2} / 4(-\Delta g)
$$

The corresponding relaxation time is

$$
\tau=\tau_{0} e^{F_{\alpha}^{\ddagger} / k_{\mathrm{B}} T}
$$

We remind the reader that in the equilibrium fluid regime, this barrier reproduces the venerable Vogel-Fulcher law. For shallow quenches, it gives the characteristic "nonlinearity" of a changed Arrhenius relaxation during aging. ${ }^{44}$ Together with eq 14 , the above result for $F^{\ddagger}$ indicates that structural relaxation events in samples quenched by compression occur more rapidly than they would in samples equilibrated at pressure $p_{\text {eq, }}$, where the only driving force is entropic: $\Delta g=-T s_{\mathrm{c}}$. This is analogous to the way a sample being slowly thermally quenched starting at temperature $T_{1}$ to the ambient temperature $T_{2}<T_{1}$, has an extra driving force for relaxation due to the enthalpic stabilization $^{44} \int_{T_{1}}^{T_{2}} \Delta C_{\mathrm{p}} d T<0$. Here, $\Delta C_{\mathrm{p}}$ stands for the configurational specific heat. Any externally imposed shear also would add to the driving force ultimately giving the yield strength of the glass. ${ }^{61}$

The cooperativity size $N^{*}$, defined by the relation $F\left(N^{*}\right)=0$ :

$$
N^{*}=\left(\frac{\gamma}{\Delta g}\right)^{2}
$$

indicates the size of a region that is guaranteed to have another aperiodic mechanically stable state. The quantity $N^{*}$ is very important because it signifies the size below which the sample is mechanically stable. In contrast, a larger region is only metastable on the time scale defined by the barrier from eq 17. Note the simple relation

$$
N^{*}=4 N^{*}
$$

How do the barrier and critical size for an aging reconfiguration event depend on the depth of the quench? In Figure 6, we show these two quantities as functions of the filling fraction in the quenched structure. Each panel of the figure exclusively pertains to barriers found from states obtained by quenching along a specific configurational adiabat, $s_{\mathrm{c}}=$ const, approximated according to eq 8 . The specific value of $\varphi_{\max }$ chosen in panel (a) refers to the Salsburg-Wood adiabat that is near but not too close to the spinodal crossover so as to avoid barrier softening effects due to spatial variations of the order parameter $\alpha{ }^{18}$ The value of $\varphi_{\max }$ chosen for plotting in panel (b) corresponds with a barrier at equilibrium that is numerically close to the typical barrier at the laboratory glass transition, i.e., $F^{\ddagger} / k_{\mathrm{B}} T=\ln \left(10^{4} \mathrm{sec} / 10^{-12} \mathrm{sec}\right) \approx 37$. Incidentally, we observe that the value of the critical size $N^{\ddagger}$ found with these equation of states is in remarkably good agreement with earlier predictions of the RFOT theory. ${ }^{42,48,62,69}$ Here and everywhere below, we parametrize the density dependence of the configurational entropy, in equilibrium, using an interpolation formula $s_{\mathrm{c}}(\varphi)=s_{\mathrm{c}}\left(\varphi_{\mathrm{cr}}\right)\left(\varphi_{\mathrm{K}}-\varphi\right) /\left(\varphi_{K}-\varphi_{\mathrm{cr}}\right)$ with $s_{\mathrm{c}}\left(\varphi_{\mathrm{cr}}\right)=$ $1.75 k_{\mathrm{B}}{ }^{27,47}$ We assume the configurational part of the entropy 
remains constant along the Salsburg-Wood adiabats, as mentioned.

We notice that for sufficiently high pressures, the barrier eventually decreases with pressure and saturates at a value that depends on the proximity of the Salsburg-Wood adiabat to the Kauzmann line, at which by construction $\varphi_{\max }=\varphi_{\mathrm{K}}^{\infty}$ :

$$
\lim _{p \rightarrow \infty} \frac{F^{*}}{k_{\mathrm{B}} T}=\frac{\varphi_{\max }}{\varphi_{\mathrm{K}}^{\infty}-\varphi_{\max }} \frac{1+\sigma}{8(1-2 \sigma)}
$$

see the Supporting Information. Here, $\varphi_{\max }$ pertains to the quench in question. The low-pressure behavior of the barriers, on the other hand, depends on the proximity of the SalsburgWood adiabats to the equilibrium crossover. For sufficiently small $\varphi_{\max }$ and low pressures, the barrier at first increases with the depth of the quench before adopting the aforementioned high-pressure trend. The increase of the thermally scaled barrier with the extent of quench is, in fact, observed in routine thermal quenches in the laboratory, consistent with the expectation from this calculation. ${ }^{18}$

It is instructive to note that in the high pressure limit, we can combine eqs $11-13$ with eq 5 to obtain

$$
\varphi_{\max } \tilde{p}-\varphi_{\mathrm{K}}^{\infty} \tilde{p}_{\mathrm{eq}}=\tilde{p}_{\mathrm{eq}}^{2}\left(\varphi_{\mathrm{K}}^{\infty}-\varphi_{\max }\right) \frac{2(1-2 \sigma)}{3(1+\sigma)}
$$

This result implies that the pressure inside the reconfigured region scales only as the square root of the external pressure:

$$
p_{\text {eq }}=p^{1 / 2}\left[\frac{\varphi_{\max }}{\left(\varphi_{\mathrm{K}}^{\infty}-\varphi_{\max }\right)} \frac{3(1+\sigma)}{2(1-2 \sigma)}\right]^{1 / 2}, p \rightarrow \infty
$$

and thus the relaxed interior pressure becomes arbitrarily smaller than the external pressure as the latter grows to be infinite. The resulting, arbitrarily large pressure discontinuity arising from the matrix' rigidity implies that the outside region is unable to relax to fill the effective void formed within the reconfigured region and, thus, is jammed in a dome-like configuration. While domes made of engineered blocks can be perfectly stable, no such stability can be expected from a dome made of frictionless spheres with finite thermal vibration. At the same time, we have seen that while the reconfiguration barrier saturates at a finite value, with increasing pressure, the interparticle forces can be made arbitrarily large, in which case finite-sized displacements of particle become virtually barrierless: If formed in this regime, the "dome" would promptly collapse from any thermal motion! Another way to look at this is that the vibrational displacement in the original quenched structure can be made arbitrarily small by sufficient external compression. As a result of the pressure mismatch, ( $p$ $\left.-p_{\mathrm{eq}}\right)$, the aging-induced displacement of the particle can then exceed this typical vibrational displacement to an arbitrarily large degree implying that the vibrational cage is destroyed by thermal motion.

We see that the critical size $N^{\ddagger}$ for compact rearrangements could become as small as one wishes, apparently, even smaller than one. (For the reader's reference, Figure 4 shows where, on the Salsburg-Wood adiabats, the critical size becomes exactly equal to 1.) This means there will be individual one-particle reconfigurations that have small barriers. Thus, a series of reconfiguration events would then occur in a spatially contiguous fashion so as to form strings and, ultimately, percolation clusters, see Figure $7 \mathrm{a}$. Indeed this means the instability threshold itself will occur with less additional driving

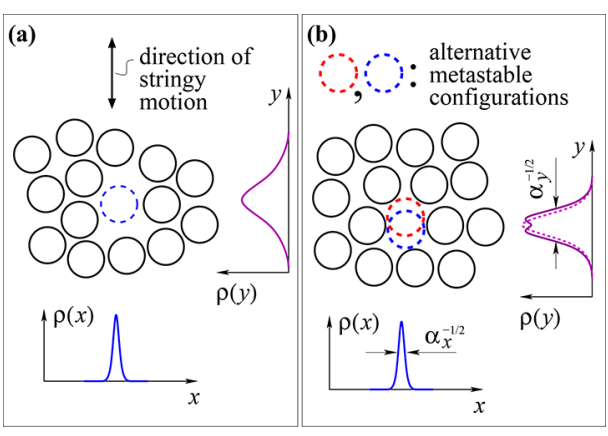

Figure 7. (a) A stringy instability is signaled by the ability of a particle to escape the cage via a displacement that significantly exceeds the typical vibrational displacement. The vibrational amplitude in the direction of lower pressure exceeds its typical value, while the collision frequency is lowered. (b) Illustration of how compression of an anisotropic cage can lead to a local symmetry breaking upon which the caged particle will be subject to a bistable effective potential and will have to choose one of the minima upon further compression, while leaving the remainder of the cage slightly undercoordinated.

force than we have computed assuming compact reconfiguration events. Stevenson, Schmalian, and Wolynes ${ }^{16}$ determined the free energy cost for forming such strings or fractal "lattice animals" when in the liquid state, i.e., for equilibrium conditions. Their analysis was later generalized by Wisitsorasak and Wolynes to accommodate for the presence of additional bulk driving forces as can occur in the glass, such as mechanical stress. ${ }^{61}$ The free energy profile for creating a string or fractal cluster, like the compact rearrangement, can be written as a function of size $N$ but contains an additional size-dependent shape entropy of the reconfigured region:

$$
F(N)=\gamma^{\prime} N+\Delta g N-k_{\mathrm{B}} T(\ln \Omega) N
$$

Here $\gamma^{\prime}$ is the extrapolated free energy cost to reconfigure one particle. Similarl to the coefficient $\gamma$ from eq 9, the quantity $\gamma^{\prime}$ reflects the cost of breaking nearly all the contacts around the particle and is a fraction of the mismatch coefficient $\gamma$ for compact clusters: ${ }^{16}$

$$
c_{\gamma} \equiv \gamma^{\prime} / \gamma<1
$$

We expect the above ratio not to be singular and remain roughly constant. The linear size-dependence of the penalty for forming a string or fractal cluster of size $N, \gamma^{\prime} N$, follows from geometry. The quantity $\Omega$ reflects the multiplicity of strings that can emanate from a given locale; its value can be adjusted to effectively account for string percolation using known properties of percolation clusters. ${ }^{16}$ Numerically, one has $k_{\mathrm{B}} \ln$ $\Omega \approx 2 k_{\mathrm{B}}$, a value we adopt here for concreteness, also gives the correct scaling of the crossover temperature with fragility. ${ }^{16}$

According to eq 24, fractal excitations can form near a spinodal once the slope of the $N$ dependence is nonpositive:

$$
\left(\gamma^{\prime}+\Delta g-k_{\mathrm{B}} T \ln \Omega\right) \leq 0
$$

For equilibrium states, the equality corresponds with the crossover density $\varphi_{\text {cr }}$ in the liquid. Below the latter density, the strings are technically infinite in length, corresponding with the uniform liquid, while for $\varphi>\varphi_{\mathrm{cr}}$ the strings are only finite and a compact region must rearrange for flow. ${ }^{16}$

In quenched states, we must determine where on the arrowed curves in Figure 4 the condition (eq 26) for marginal stability is satisfied since the driving force depends on the amount of contraction. It follows that the highest density at 
which the condition (eq 26) is still satisfied is given by the equation:

$$
\tilde{\gamma}^{\prime}=\tilde{p}^{(\mathrm{s})}-\tilde{p}_{\mathrm{eq}}+s_{\mathrm{c}} / k_{\mathrm{B}}+\ln \Omega+\Delta s_{\text {vibr }}
$$

where we used eqs 14 and 15. The label "(s)" signifies "spinodal," in reference to the point of marginal stability.

As before, pairs of a quenched state and a corresponding relaxed state are determined by simultaneous solution of eqs 11 and (12). The loci $\tilde{p}^{(s)}(\varphi)$, that result from the calculation for hard spheres, are plotted as the "string spinodal" line in Figure 4. Note that at the equilibrium crossover, where there is only an entropic force, the aforementioned parametrizations $s_{\mathrm{c}}\left(\varphi_{\mathrm{cr}}\right)=$ $1.75 k_{\mathrm{B}}$ and $k_{\mathrm{B}} \ln \Omega \approx 2 k_{\mathrm{B}}$ for our equations of state determine the appropriate numerical value of $c_{\gamma}$, which takes on a reasonable value of 0.49 .

As suggested by Figure 4, the high-pressure end of the stringspinodal line asymptotes to the infinite-pressure states that are quenched from the Kauzmann point. This is indeed the case; the corresponding scaling form can be straightforwardly determined and is not affected by the precise parametrization of $s_{\mathcal{c}}, \ln \Omega$, and $\Delta s_{\text {vibr }}$ but only the high-pressure asymptotics of the shear modulus, see the Supporting Information for explicit expressions. The Supporting Information also provides an expression for the slope of the string-spinodal line, near the equilibrium crossover, that uses experimentally measurable materials constants. ${ }^{26,27,47,70,71}$ Our estimates indicate that just as for hard spheres, the low pressure end of the spinodal line in molecular substances should have a steeper slope, in the $(\varphi$, $p^{-1}$ ) plane, than the equation of state for a configurationally adiabatic quench starting at the equilibrium crossover. We remind the reader that owing to fluctuation and the emergence of activated dynamics, the spinodal line signifies a gradual crossover, not a sharp boundary. The line also represents a glass transition since on its high-density side, the dynamics are activated and depend on the time scale of observation and preparation.

The spinodal appears as a mechanical instability of the apparently rigid state when coming from the high density side to lower densities. String-mediated aging events locally destroy the existing cages in an avalanche-like fashion. When approached from the low-density, replica-symmetric side, however, the same line can be viewed as signaling the onset of effective rigidity percolation, as pointed out in the Introduction. After the spinodal line is crossed, activation barriers begin to rise rapidly and the system typically will fall out of equilibrium on a fixed observation time scale. The density dependence of these barriers, for the five specific aging paths from Figure 4, are shown in Figure 8. The corresponding cooperativity sizes $N^{*}$, eq 19, are shown in Figure 9. For the reader's reference, we have indicated the location of the string instability, for each respective curve, with a purple asterisk. In a complete treatment, one should include barrier softening effects, which would then show both the computed barrier and cooperativity size vanishing in a critical fashion at the spinodal. The softening effects stem from spatial variations of the local order parameters and effectively erase the activated barrier at the spinodal, so the liquid motions are slowed exclusively owing to mode-coupling effects or other effects such as the viscous drag of colloidal particles against the solvent. Away from the spinodal, the barrier-softening effects become increasingly less important, see quantitative estimates for specific substances in ref 44 . In any event, a parametric plot

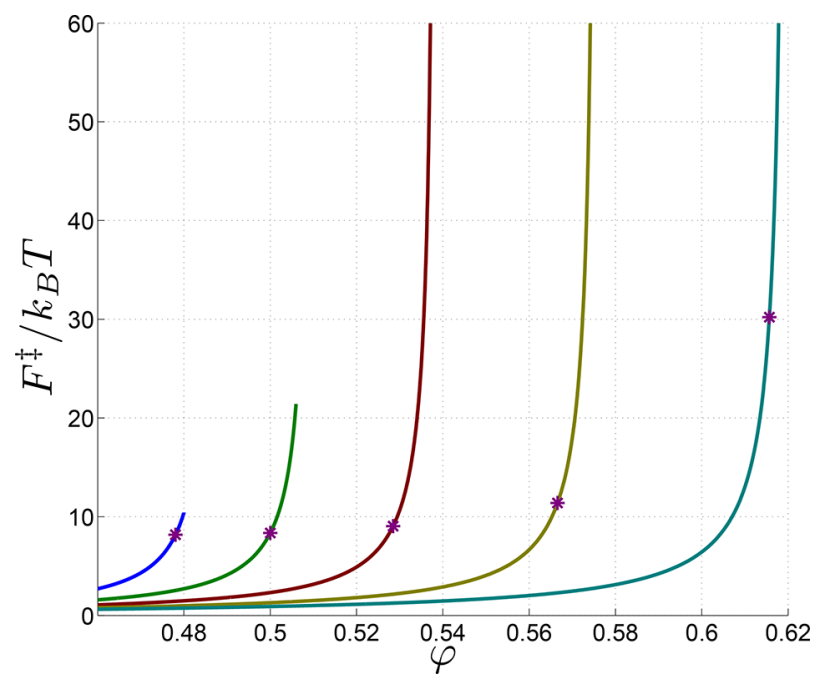

Figure 8. Density dependence of the dimensionless activation barrier from eq 17 along the five local-collapse paths indicated by the arrowed curves in Figure 4. The asterisks denote the predicted location of the spinodal from the string theory argument. The expression eq 17 does not include the barrier-softening effects near the spinodal. ${ }^{44}$ In a more complete calculation, the activation barrier would vanish at the densities indicated by the asterisks.

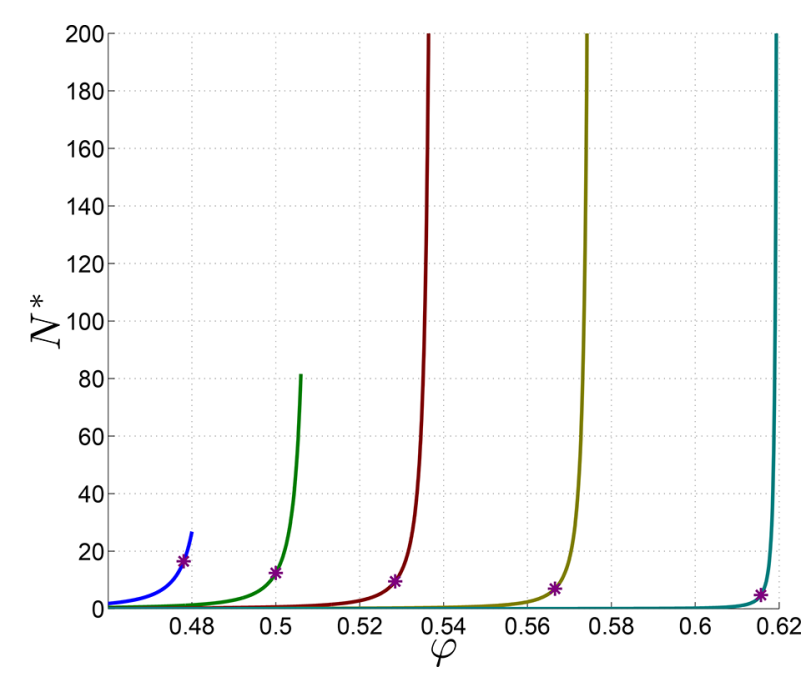

Figure 9. Density dependence of the cooperativity size $N^{*}$ of compact clusters from eq 19 along the five local-collapse paths indicated by the arrowed curves in Figure 4. The asterisks denote the location of the string spinodal. Again, no softening effects are included. ${ }^{44}$ If softening were included, the appropriate cooperativity size near the spinodal would correspond to the typical string length and would diverge except for string overlap effects.

of the activation barrier $F^{+}$vs the cooperativity size of compact clusters $N^{*}$ is expected to be insensitive to softening effects. Such a parametric plot, shown in Figure 10, indicates that the onset of activation, upon crossing the spinodal line from the low-density side, gradually changes in character with increasing pressure. At a fixed value of the activation barrier-which corresponds to a fixed observation time up to the prefactor $\tau_{0}$ in eq 18 , the initial relaxation events span fewer particles the higher the pressure at which the spinodal was reached. This reflects the aforementioned notion that at a fixed density, higher pressure corresponds with a smaller coordination 


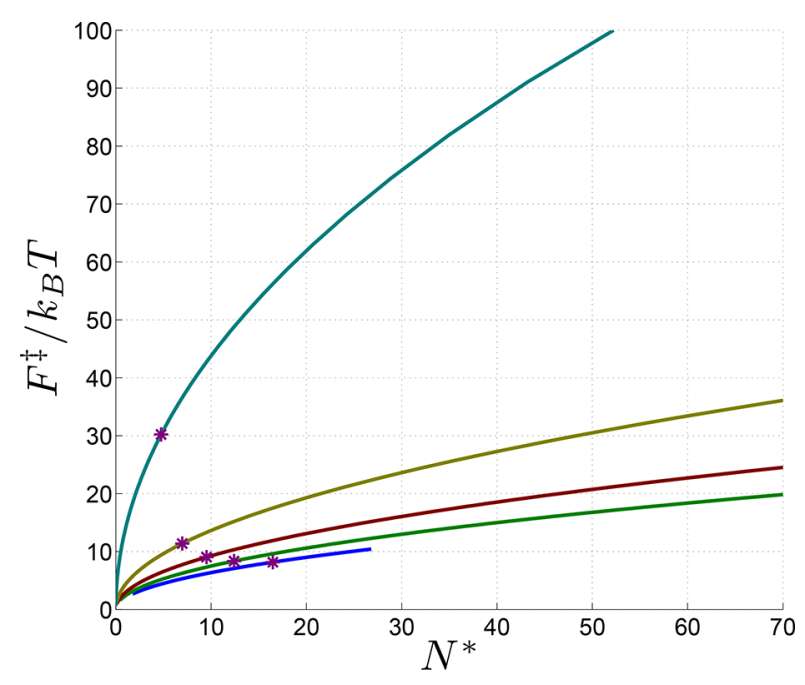

Figure 10. Parametric plot of the dimensionless barrier $F^{\ddagger} / k_{\mathrm{B}} T$ vs the compact cluster cooperativity size $N^{*}$ from Figures 8 and 9 along the five local-collapse paths indicated by the arrowed curves in Figure 4 . The asterisks denote the location of the predicted string spinodal.

number and, hence, relatively open structures. Such open structures can reconfigure by moving relatively few particles.

One concludes that a colloidal suspension that is quenched starting from a low density will reach the string-spinodal line from the left-hand side on the diagram in Figure 4 through a series of low barrier, string-like relaxations. Once the boundary where there can be a stable aperiodic crystal state is reached, relaxation events abruptly begin to slow down because they now require activation. The barrier increase will dramatically extend the lifetime of the metastable structure compared with the already long time scales needed for colloidal particles to move about. On times scales shorter than the so extended time, the system will appear to be arrested and, in fact, will appear to be jammed since the thermal forces are small relative to the forces that are imposed externally.

Following an activated reconfiguration event, our argument for the stress profile suggests the immediate surroundings of the reconfigured region will experience a partial increase in shear stress. The magnitude of the stress then falls off as $1 / r^{3}$ as one moves away from the rearranging region. Wisitsorasak and Wolynes $^{61}$ have shown that externally imposed shear on glasses leads to an instability. This argument gives quantitative values for the yield strength of glasses. Thus, we see that if a local aging event occurs in a system that is on the high density side of the crossover line but not too far away from it, the surrounding regions will in turn become unstable to forming additional fractal excitations thus facilitating a series of avalanches nearby. After reconfiguration, the newly equilibrated regions, now denser than they were before, will lead to force percolation. Detailed analysis of these facilitation effects will be a subject of future work.

\section{RELATION TO LOCAL BUCKLING INSTABILITY}

We cannot emphasize enough that the relaxation phenomena we have discussed in this paper are not accessible to strictly meanfield analyses for two distinct reasons: On the one hand, relaxation events are local with finite barriers. On the other hand, the relaxations are truly irreversible and result in either a spatially compact, activated reconfiguration from a metastable aperiodic free energy minimum to a stable aperiodic minimum or a nearly barrierless process upon which the system then again finds itself locally in a stable free energy minimum. For these reasons, the aging relaxations have no analog when $D=$ $\infty$, where all particles are treated equivalently. Nevertheless, this does not necessarily mean that marginal stability effect similar to those inferred for meanfield liquids could not be observed in finite dimensions.

Because the local environment in an aperiodic structure does not typically satisfy any point symmetries, one expects that local cages in an aperiodic crystal should be less isotropic than they are in periodic crystals. Suppose for the sake of argument that the cage for a spherical particle is somewhat elongated in one direction. Notice this would involve endowing the scalar $\alpha$ with an additional component that is a second rank tensor. ${ }^{72,73}$ As graphically illustrated in Figure $7 \mathrm{~b}$, the particle-particle gap along the short axes of the cage can be made arbitrarily smaller than the gaps to particles along the longer axis. This implies that the particle in question would be considered as making contact with only a fraction of its original neighbors, if jammed to high pressure. If the resulting number of contacts is less than that required for mechanical stability, the particle's being in the middle of the cage corresponds to an unstable, hypostatic configuration. It follows that the latter unstable configuration will itself be a transition state separating two stable configurations, one of which will be inevitably chosen thus implying a symmetry breaking transition. This transition will be accompanied by the formation of a region with modestly less coordination on the other end of cage. This cage "buckling" can be thought of as the initiation of a stringy or fractal reconfiguration event. A closely related but somewhat different view of how reconfiguration events are initiated has been put forward in a series of papers by Wyart and co-workers. ${ }^{74-76}$

A qualitative argument can be made, see the Supporting Information, for the amount of local anisotropy to be expected in the local cage. This anisotropy depends on the shear modulus $\tilde{\mu}_{f}$ of the glass in the beginning of the quench. The typical spacing between particles can be expressed through its bulk modulus, as in eq 8 . As we describe in the Supporting Information, by comparing longitudinal and transverse displacements one can write down the following, qualitative criterion for when cages made of the nearest neighbors would begin to buckle:

$$
\tilde{K} \simeq(9 \pi / 2) \tilde{\mu}_{\mathrm{f}}
$$

The solution for this equation allows us to plot a one-particle "buckling instability" line in Figure 4. We notice this line crosses the "Kauzmann line" $s_{c}=0$ at a finite pressure.

Likewise, larger regions could also undergo higher-order buckling transitions when we think of a region having to choose between two alternative states. Such transitions would however occur at higher pressures than those causing one-particle buckling instabilities:

$$
\tilde{K} \gtrsim \tilde{\mu}_{\mathrm{f}}(L / a)^{3}
$$

where $L$ is the size of the region undergoing the symmetry breaking.

Buckling transitions, which we can see are related to the reconfiguration events discussed above-clearly result in a splitting of the original free energy basin into sub-basins. They seem to be the analog of the higher-order RSB seen in the $D=$ $\infty$ models. This suggests that the buckling is a finitedimensional analog of the Gardner transition. 


\section{FLUCTUATIONS AND DYNAMICAL HETEROGENEITY}

We have analyzed the activated events and instabilities in the amorphous solids in an approximation that accounts for the exponential multiplicity of glassy aperiodic crystals but that neglects the fluctuations in specific volume, energy, and configurational entropy that inevitably arise from this diversity. $\mathrm{Xia}$ and Wolynes ${ }^{77}$ showed how the fluctuations in driving force for activated transitions can account for dynamical heterogeneity. Their analysis predicted, in quantitative agreement with experiment, the correlation between the stretching exponent for the alpha relaxation in equilibrated glassy liquids and liquid fragility. Stevenson and Wolynes later showed how these driving-force fluctuations also act to smear out the instabilities due to fractal excitations. This smearing then gives rise to a tail to the barrier distribution referred to as the secondary or beta relaxations. Their theory also predicts that beta relaxation events have merged with the alpha relaxation near the crossover point.

In the moderate pressure regime for amorphous solids these earlier analyses should suffice but since at high external pressure the driving force also has a contribution from the volume mismatch energy, the fluctuations of the latter need to be accounted for at high pressures. These fluctuations should depend on fluctuations in specific volume and shear modulus. We leave further analysis of the fluctuation effects, owing to their complexity, to future work. We note that fluctuations in the driving force can, if large enough, completely destroy the random first order transition itself and turn it into a continuous one. This was discussed by Stevenson et al. ${ }^{14}$ in their explicit mapping of glassy liquid landscapes onto magnets with both random fields and random couplings. Near equilibrium for molecular liquids, their analysis predicted that the one step RSB transition remains intact. The fluctuations in driving force, in principle, however could become large enough to destroy the 1RSB transition at large pressures. To get a sense of this effect, we note that by eqs 14 and 5 fluctuations in the driving force at the cooperativity size $N^{*}$ can be roughly estimated as $\delta \Delta g \simeq p / \sqrt{3 N^{*}}$, where we have used the standard expression $^{67}$ for pressure fluctuations: $(\delta p)^{2}=k_{\mathrm{B}} T K_{\mathrm{S}} / V$, where $K_{\mathrm{S}}$ is the adiabatic bulk modulus and $V$ volume. Clearly, even for the smallest allowable values of $N^{*}$ of order one, $\delta \Delta g<|\delta \Delta g|$. This implies that fluctuations in the driving force should modify the energetics of activation only quantitatively, not qualitatively. In any event, the present argument leaves open the possibility of an additional region of marginal stability at high pressures near the densest random-close packing, see also our earlier remarks regarding the buckling-instability line.

\section{SUMMARY AND DISCUSSION}

To zeroth order, amorphous solids can be thought of as being quenched liquids. But when we look more deeply, we see that their properties do depend on the way they are prepared. In this regard it is important to realize that on our diagram plotted in terms of dimensionless quantities, the quenching protocols are typically quite different for making molecular glasses than they are for preparing amorphous solids made of larger particles such as colloids or grains. Ultimately these differences trace back to the enormous size differences between atoms, colloidal particles, and grains. Molecular glasses, owing to their being made of rapidly moving atomic-scale constituents, become sensibly rigid and fall out of equilibrium in the laboratory only deep in the activated regime, when the activation barriers are quite high, leading to times 16 orders of magnitude beyond the basic molecular scale. In contrast, the intrinsic thermal motions of colloids or granular objects are already quite slow even when unactivated because the particles are so large. This means the emergence of even a very modest barrier is enough to lead to apparent rigidity on human time scales. Another contrast between molecular glasses and colloidal assemblies is that the hard-sphere, or kinetic, part of the pressure is already very large for molecular liquids before they are quenched but in the total pressure, this hard sphere contribution is nearly canceled by a very large attractive contribution due to interparticle cohesive forces. ${ }^{50}$ Thus, very high dimensionless kinetic pressures are very difficult to achieve for molecular liquids by compression in the laboratory. They typically require at least megapascals to have any significant effects; such pressures require specialized apparatus. For colloids or granular assemblies, in contrast, there is little problem achieving, in the laboratory, very high dimensionless kinetic pressures because the absolute number density of the constituents of such assemblies is orders of magnitude lower in absolute terms, so the compression energy is easy to maintain. These differences between molecular and colloidal systems mean that the compression effects highlighted in this paper hardly show up in laboratory studies or molecular glasses but become paramount for experiments on granular assemblies or colloids. The high compression possible for the colloids or granular assemblies means that "jamming" occurs quite readily for them but does not occur to any extent for molecular glasses. At the same time compression still can only barely take a typical colloidal system into the activated region. This implies amorphous solids prepared from grains or colloids inevitably end up being near the line of marginal stability but molecular glasses prepared in the laboratory will generally not be marginally stable but will be deep in the activated regime.

The string-induced spinodal apparently could a priori lead to a divergent correlation length and, hence, criticality. Any incipient criticality however would be avoided because of the local activated events. Indeed, already the dimensionless activation barrier $F^{\ddagger} / k_{\mathrm{B}} T$ decreases with pressure for a sample quenched starting in the landscape regime, as does of course the relaxation time, which scales exponentially with $F^{\ddagger} / k_{\mathrm{B}} T$. (There is also a decrease in the prefactor because the collisional time decreases with pressure as $1 / p$.) We see that activated events should destroy long-range correlations in the nonequilibrium solid entirely just as the incipient criticality due to mode-mode coupling effects is destroyed by the activated reconfigurations in equilibrated liquids..$^{17,18,78}$ It is relevant to compare a recent discussion in ref 79 of the role of activated and avalanche events in the random field Ising magnet, which we have emphasized earlier has many close connections with the RFOT picture.

That the incipient long-range correlations are short-circuited by activated reconfiguration events is consistent with observations on colloidal suspensions, ${ }^{80}$ where it has been possible to identify large, spatially correlated clusters that percolate the sample but only on short time scales. On times beyond a certain threshold, the clusters do break up. The threshold time can be made arbitrarily longer than the observation time by sufficiently compressing the suspension, in agreement with the results of the present analysis for the emergence of activated barriers upon crossing the string spinodal. Clearly, a rigidity-percolated colloidal suspension 
can be regarded as jammed because its temperature is effectively zero.

We note that our view of jamming phenomena as a spinodal is consistent with "unjamming" simulations of soft repulsive spheres due to Ikeda et al., ${ }^{81}$ who found a scaling relation between the correlation length $\xi_{j}$, relaxation time $\tau_{j}$, and proximity $\left|\varphi_{j}-\varphi\right|$ to an unjamming transition. At sufficiently low temperatures, they find $\xi_{j}^{2} \propto \tau_{j} \propto\left|\varphi_{j}-\varphi\right|^{-1 / 2}$. Now, in general one finds near a meanfield spinodal, $\xi_{j} \sim\left|\varphi_{j}-\varphi\right|^{-1 / 4}$. ${ }^{2}$ Kirkpatrick and Wolynes identified this spinodal scaling in their early analysis of glasses in the Kac limit. ${ }^{17}$ Also assuming no dynamical renormalization, the Onsager-Landau ansatz for the evolution of an order parameter, say $\eta: \dot{\eta} \propto \partial \mathrm{F} / \partial \eta,{ }^{83}$ leads to $\tau_{j}$ being proportional to $\xi_{j}^{2}$ ( $F$ is the appropriate free energy). This is just what Ikeda et al. ${ }^{81}$ found.

Colloidal suspensions differ in an essential way from molecular liquids in that their dimensionless kinetic pressure is much lower than the dimensionless pressures that is ordinarily applied by the experimenter. (This fact is often referred to as such a suspension being nearly zero temperature, a notion that is even more pertinent to granular matter.) Accordingly, the collisional time scale, which is now set by the viscosity of the solvent and the very large particle size, is so long that configurational equilibration is intrinsically much slower than in molecular-scale glassformers, easily by 10 orders of magnitude. ${ }^{8}$ Thus, we conclude that colloidal suspensions will typically approach the string crossover from the low density side, where they become solidified. Upon compression, the suspension begins to relax toward high density states at an (osmotic) pressure greatly exceeding its equilibrium value (but still much lower than experimenter-inflicted forces). Until the string-crossover line is reached, relaxations are essentially barrierless, being limited primarily by the viscous drag of the solvent on the individual colloidal particles. During such relaxation, individual particles will move distances comparable to the particle spacing. However, once the crossover is reached, there will now arise activation barriers, ${ }^{18}$ which will further slow down the relaxation, while the particle displacements will become significantly smaller and comparable to the typical vibrational amplitude. The latter amplitude decreases inversely proportionally with the pressure, as already mentioned. Because of the emergence of activation, the suspension will be largely arrested on experimentally relevant scales beyond but very near to the spinodal line.

The above discussion of colloidal suspensions is also directly relevant to computer simulations of hard spheres that are monodisperse or nearly so. In this case, the "experimenter's" time scale must again be very short out of necessity because monodisperse hard spheres actually crystallize readily. Thus, like colloidal systems in the laboratory, simulations of monodisperse hard spheres leave them near the marginality line (with its accompanying isostatic $z$ ) since activated aging takes too much time. On the other hand, heterodisperse mixtures do not crystallize as easily as do monodisperse hard spheres, which may allow simulations to go somewhat deeper in the activated regime implying relatively high pressures are needed for the consequences of marginality to show up, see below. One caveat is that it is not clear at present to what extent the slow processes in such polydisperse mixtures could entail phase separation and demixing. The latter effects seem to be particularly important in continuously heterodisperse mixtures, whose mixing entropy is strictly infinite, see however ref 84 . We leave this aspect of the problem for future work.
We have mentioned a way of looking at the symmetry breaking taking place in quenched glasses as a "buckling transition." Conceivably from this viewpoint, the low-density, replica-symmetric and high-density, 1-stage RSB region will be separated by a distinct set of instabilities that formally correspond with higher-than-one state RSB. Despite the poetic similarity of the buckling instability with the meanfield Gardner transition, the connection between the two, if any, is not yet clear. In any event, it is quite reasonable that at the spinodal between the replica-symmetric and 1-step RSB sectors, there is a potential for a continuous replica-symmetry breaking. The latter notion is consistent with the findings by Dzero et al. ${ }^{65,85}$ that interfaces between distinct aperiodic free energy minima exhibit higher-order RSB. Indeed, similar interfaces are expected to separate replica symmetric and 1-stage RSB regions near the equilibrium crossover. $^{48}$ Such interfaces exhibit significant variations in the local value of the order parameter $\alpha,{ }^{18}$ which are also requisite for the buckling instabilities. As a corollary of the above considerations-and consistent with meanfield results, we conclude that the spinodal line can also be thought of as a line of jamming-unjamming transitions. It is possible that the argument for the buckling instability line could be extended all the way to the equilibrium crossover, while its separation from the spinodal becomes progressively smaller on approach to $\varphi_{\mathrm{cr}}$. Just as for the stringy spinodal, buckling is not expected to cause true criticality owing to the activated reconfiguration events.

As pointed out in the beginning of the Section, ordinary glasses made of organic molecules or inorganic network materials-as opposed to collections of macromolecular or colloidal assemblies-are generally quenched starting well in the landscape regime. At the same time, we have seen that in order to observe the re-emergence of the string spinodal at high pressure, following a conventional quench, one must increase the kinetic pressure by several fold. Elementary estimates ${ }^{8}$ show that the kinetic pressure in actual substances, $p \simeq k_{\mathrm{B}} T / a^{2} d$, exceeds atmospheric pressure by about 4 orders of magnitude. This corresponds to Gigapascals. Such pressures have been achieved in studies of organic glassformers ${ }^{86}$ and we hope that further experiments along these lines will be performed. According to our analysis, however, to see the compressioninduced instability requires this pressurization to be sufficiently fast to avoid aging toward more stable structures. Once such aging occurs, still higher pressures are required to observe the spinodal coming from the high density, glassy side. In any event, the spinodal obviously cannot be reached using conventional thermal quenches because in the latter, the ambient pressure remains comparable to atmospheric pressure, not the Gigapascals requisite for achieving the spinodal near ordinary temperatures. This difficulty of observing marginal stability comes exclusively from the attractive interactions in actual molecular systems positioning them already quite high in the dimensionless kinetic pressure.

Although our arguments were explicitly illustrated in this paper using hard monodisperse spheres, arguments like those presented here are also applicable to soft particles, such as actual molecules or the soft repulsive spheres mentioned earlier in the context of studies of unjamming transitions by Ikeda et al. ${ }^{81}$ Equations of state for molecular substances can be mapped onto those for hard spheres; see the Supporting Information for a qualitative discussion of correlation between the slope of configurationally adiabatic quenches and the fragility of the liquid. We have already mentioned that the slope of the string 
spinodal near the equilibrium crossover is expected to be greater for molecular liquids than for strictly hard objects. This implies that reaching the spinodal instability from the landscape side in such liquids is even harder for systems with soft repulsion than for hard spheres, in light of the aforementioned difficulties of rapidly creating and then sustaining, in the laboratory, high pressures in molecular systems. When dealing with molecules, quantum effects could play a role; these effects depend on the absolute particle masses, of course. Such quantum effects are not expected to affect significantly the location of the string spinodal. Indeed, no quantities entering the condition (eq 27) explicitly refer to the particle mass except the vibrational free energy difference, which would have both an entropic part and a zero energy contribution. The vibrational free energy change during isothermal aging, which was dropped from eq 27 in the first place, will be negligible. We note that marginal stability due to buckling will be also efficiently suppressed by the quantum effects, see the Supporting Information. Finally, at the high pressures requisite for marginal stability various structural transitions of electronic origin generally begin to occur. ${ }^{87}$ These are beyond the scope of the present paper.

In view of the virtual inaccessibility of the pressure-induced spinodal in actual molecular systems and the suppression of the buckling owing to quantum effects, we conclude that strict marginal stability like that manifest in the Gardner transition is not the cause of the anomalies seen in cryogenic glasses, viz., the two-level systems and their higher-temperature companion, the Boson peak. Their universality, ${ }^{39}$ emphasized early on by $\mathrm{Yu}$ and Leggett, ${ }^{40}$ instead has its origin in the low barrier tail of both compact and stringy reconfiguration events characteristic of glassy systems in three dimensions, as we argued quite a while ago. ${ }^{41-43}$ In that early work, we showed that the universality results, in part, from an intrinsic connection between the cohesive energy of the solid and the interaction strength of the phonons with local degrees of freedom. Interestingly, this intrinsic connection itself stems from the marginal stability of one-particle motions against vibrational excitations of the lattice when the (finite frequency) rigidity sets in an equilibrated liquid. As emphasized above, these same low barrier motions destroy any incipient criticality associated with the spinodal.

It would be interesting to reach the spinodal from the high density side. One way to do this would be to study a colloidal suspension of nanoclusters of appropriate size or, perhaps, artificial polymers or biomolecules so that the intrinsic time scale of motion is neither too short nor too long. Unfortunately, nanoclusters in this size range have rather complicated particle-particle interactions. The unavoidably large dispersion forces must be compensated by Coulomb repulsion in order to avoid aggregation, which itself leads already to rather complicated phase behaviors, ${ }^{88}$ such as the formation of gels. ${ }^{89}$ Yet certain proteins, such as hemoglobin, conceivably may fit the bill.

\section{ASSOCIATED CONTENT}

\section{S Supporting Information}

The Supporting Information is available free of charge on the ACS Publications website at DOI: 10.1021/acs.jpcb.7b09553.

Details of several derivations, an extended discussion of the buckling instability, and a qualitative discussion of the slope discontinuity between the equilibrium and configurationally adiabatic equations of state (PDF)

\section{AUTHOR INFORMATION}

\section{Corresponding Author}

*vas@uh.edu.

ORCID

Vassiliy Lubchenko: 0000-0001-6398-261X

Notes

The authors declare no competing financial interest.

\section{ACKNOWLEDGMENTS}

We are privileged to dedicate this article to Ben Widom. Ben's science has been an inspiration to generations of theorists. Equally inspiring, his kindness to younger scientists has stood out as a beacon in our field. We gratefully acknowledge early discussions with Dr. Pyotr Rabochiy. V.L.'s work is supported by the NSF Grants CHE-1465125 and MCB-1518204 and the Welch Foundation Grant E-1765. P.G.W.'s work is supported by the Center for Theoretical Biological Physics sponsored by the National Science Foundation (NSF Grants PHY-1427654). Additional support to P.G.W. was provided by the D.R. BullardWelch Chair at Rice University (Grant C-0016).

\section{REFERENCES}

(1) Vitruvius. The Ten Books on Architecture; Dover Publications: New York, 1960.

(2) Maxwell, J. C. On the calculation of the equilibrium and stiffness of frames. Philos. Mag. 1864, 27, 294-299.

(3) Morice, P. The new look in structural analysis: a historical survey. Concrete 1969, 3, 415-417.

(4) Phillips, J.; Thorpe, M. Constraint theory, vector percolation and glass formation. Solid State Commun. 1985, 53, 699-702.

(5) Liu, A. J.; Nagel, S. R.; van Saarloos, W.; Wyart, M. In Dynamical Heterogeneities in Glasses, Colloids, and Granular Media; Berthier, L., Biroli, G., Bouchaud, J.-P., Cipelletti, L., van Saarloos, W., Eds.; Oxford University Press: Oxford, 2011; pp 1-44.

(6) DeGiuli, E.; Laversanne-Finot, A.; During, G.; Lerner, E.; Wyart, $M$. Effects of coordination and pressure on sound attenuation, boson peak and elasticity in amorphous solids. Soft Matter 2014, 10, 56285644.

(7) Lubchenko, V.; Wolynes, P. G. Theory of Structural Glasses and Supercooled Liquids. Annu. Rev. Phys. Chem. 2007, 58, 235-266.

(8) Lubchenko, V. Theory of the structural glass transition: A pedagogical review,. Adv. Phys. 2015, 64, 283-443.

(9) Kauzmann, W. The nature of the glass state and the behavior of liquids at low temperatures,. Chem. Rev. 1948, 43, 219-256.

(10) Charbonneau, P.; Jin, Y.; Parisi, G.; Rainone, C.; Seoane, B.; Zamponi, F. Numerical detection of the gardner transition in a meanfield glass former. Phys. Rev. E 2015, 92, 012316.

(11) Singh, Y.; Stoessel, J. P.; Wolynes, P. G. The hard sphere glass and the density functional theory of aperiodic crystals,. Phys. Rev. Lett. 1985, 54, 1059-1062.

(12) Stoessel, J. P.; Wolynes, P. G. Linear excitations and stability of the hard sphere glass. J. Chem. Phys. 1984, 80, 4502-4512.

(13) Kirkpatrick, T. R.; Thirumalai, D.; Wolynes, P. G. Scaling concepts for the dynamics of viscous liquids near an ideal glassy state. Phys. Rev. A: At., Mol., Opt. Phys. 1989, 40, 1045-1054.

(14) Stevenson, J. D.; Walczak, A. M.; Hall, R. W.; Wolynes, P. G. Constructing explicit magnetic analogies for the dynamics of glass forming liquids. J. Chem. Phys. 2008, 129, 194505.

(15) Unger, C.; Klein, W. Nucleation theory near the classical spinodal. Phys. Rev. B: Condens. Matter Mater. Phys. 1984, 29, 26982708. 
(16) Stevenson, J. D.; Schmalian, J.; Wolynes, P. G. The shapes of cooperatively rearranging regions in glass-forming liquids. Nat. Phys. 2006, 2, 268-274.

(17) Kirkpatrick, T. R.; Wolynes, P. G. Stable and metastable states of mean-field Potts and structural glasses. Phys. Rev. B: Condens. Matter Mater. Phys. 1987, 36, 8552-8564.

(18) Lubchenko, V.; Wolynes, P. G. Barrier softening near the onset of nonactivated transport in supercooled liquids: Implications for establishing detailed connection between thermodynamic and kinetic anomalies in supercooled liquids. J. Chem. Phys. 2003, 119, 90889105.

(19) Bhattacharyya, S. M.; Bagchi, B.; Wolynes, P. G. Bridging the gap between the mode coupling and the random first order transition theories of structural relaxation in liquids. Phys. Rev. E 2005, 72, 031509.

(20) Johari, G. P.; Goldstein, M. Viscous liquids and the glass transition. II. Secondary relaxations in glasses of rigid molecules. J. Chem. Phys. 1970, 53, 2372-2388.

(21) Gross, D. J.; Kanter, I.; Sompolinsky, H. Mean-field theory of the potts glass. Phys. Rev. Lett. 1985, 55, 304-307.

(22) Lopatin, A. V.; Ioffe, L. B. Structural glass on a lattice in the limit of infinite dimensions. Phys. Rev. B: Condens. Matter Mater. Phys. 2002, 66, 174202.

(23) Wu, S. W.; Westfahl, H.; Schmalian, J.; Wolynes, P. G. Theory of microemulsion glasses. Chem. Phys. Lett. 2002, 359, 1-7.

(24) Ashcroft, N. W.; Mermin, N. D. Solid State Physics; Harcourt Brace College Publishers: Fort Worth, 1976.

(25) Fixman, M. Highly anharmonic crystal. J. Chem. Phys. 1969, 51, $3270-3279$.

(26) Lowen, H. Elastic constants of the hard-sphere glass: a density functional approach. J. Phys.: Condens. Matter 1990, 2, 8477-8484.

(27) Rabochiy, P.; Lubchenko, V. Universality of the onset of activated transport in Lennard-Jones liquids with tunable coordination: Implications for the effects of pressure and directional bonding on the crossover to activated transport, configurational entropy and fragility of glassforming liquids. J. Chem. Phys. 2012, 136, 084504.

(28) Berthier, L.; Charbonneau, P.; Jin, Y.; Parisi, G.; Seoane, B.; Zamponi, F. Growing timescales and lengthscales characterizing vibrations of amorphous solids. Proc. Natl. Acad. Sci. U. S. A. 2016, $113,8397-8401$.

(29) Charbonneau, P.; Kurchan, J.; Parisi, G.; Urbani, P.; Zamponi, F. Fractal free energy landscapes in structural glasses. Nat. Commun. 2014, 5, 3725 .

(30) Mari, R.; Krzakala, F.; Kurchan, J. Jamming versus glass transitions. Phys. Rev. Lett. 2009, 103, 025701.

(31) Mari, R.; Kurchan, J. Dynamical transition of glasses: From exact to approximate. J. Chem. Phys. 2011, 135, 124504.

(32) Kurchan, J.; Parisi, G.; Urbani, P.; Zamponi, F. Exact theory of dense amorphous hard spheres in high dimension. ii. the high density regime and the gardner transition. J. Phys. Chem. B 2013, 117, 1297912994.

(33) Gardner, E. Spin glasses with p-spin interactions. Nucl. Phys. B 1985, 257, 747-765.

(34) Rainone, C.; Urbani, P.; Yoshino, H.; Zamponi, F. Following the evolution of hard sphere glasses in infinite dimensions under external perturbations: Compression and shear strain. Phys. Rev. Lett. 2015, $114,015701$.

(35) Urbani, P.; Biroli, G. Gardner transition in finite dimensions. Phys. Rev. B: Condens. Matter Mater. Phys. 2015, 91, 100202.

(36) Scalliet, C.; Berthier, L.; Zamponi, F. Absence of marginal stability in a structural glass. Phys. Rev. Lett. 2017, 119, 205501.

(37) Amorphous Solids: Low-Temperature Properties; Phillips, W. A., Ed.; Springer-Verlag: Berlin, Heidelberg, NY, 1981.

(38) Tunneling Systems in Amorphous and Crystalline Solids; Esquinazi, P., Ed.; Springer-Verlag, Heidelberg, 1998.

(39) Freeman, J. J.; Anderson, A. C. Thermal conductivity of amorphous solids. Phys. Rev. B: Condens. Matter Mater. Phys. 1986, 34, 5684-5690.
(40) Yu, C. C.; Leggett, A. J. Low temperature properties of amorphous materials: Through a glass darkly. Comments Cond. Mater. Phys. 1988, 14, 231-251.

(41) Lubchenko, V.; Wolynes, P. G. The microscopic quantum theory of low temperature amorphous solids,. Adv. Chem. Phys. 2007, 136, 95-206.

(42) Lubchenko, V.; Wolynes, P. G. Intrinsic quantum excitations of low temperature glasses. Phys. Rev. Lett. 2001, 87, 195901.

(43) Lubchenko, V.; Wolynes, P. G. The origin of the boson peak and thermal conductivity plateau in low temperature glasses. Proc. Natl. Acad. Sci. U. S. A. 2003, 100, 1515-1518.

(44) Lubchenko, V.; Wolynes, P. G. Theory of aging in structural glasses. J. Chem. Phys. 2004, 121, 2852-2865.

(45) Gebremichael, Y.; Vogel, M.; Glotzer, S. C. Particle dynamics and the development of string-like motion in a simulated monoatomic supercooled liquid. J. Chem. Phys. 2004, 120, 4415-4427.

(46) Stevenson, J. D.; Wolynes, P. G. The ultimate fate of supercooled liquids. J. Phys. Chem. A 2011, 115, 3713-3719.

(47) Rabochiy, P.; Lubchenko, V. Liquid state elasticity and the onset of activated transport in glass formers. J. Phys. Chem. B 2012, 116, $5729-5737$.

(48) Xia, X.; Wolynes, P. G. Fragilities of liquids predicted from the random first order transition theory of glasses. Proc. Natl. Acad. Sci. U. S. A. 2000, 97, 2990-2994.

(49) Mézard, M.; Parisi, G. In Structural Glasses and Supercooled Liquids: Theory, Experiment, and Applications; Wolynes, P. G., Lubchenko, V., Eds.; John Wiley \& Sons: Hoboken, NJ, 2012; pp $151-191$.

(50) Longuet-Higgins, H. C.; Widom, B. A rigid sphere model for the melting of argon. Mol. Phys. 1964, 8, 549-556.

(51) Hansen, J. P.; McDonald, I. R. Theory of Simple Liquids; Academic Press: New York, 1976.

(52) Andersen, H. C.; Weeks, J. D.; Chandler, D. Relationship between the hard-sphere fluid and fluids with realistic repulsive forces. Phys. Rev. A: At., Mol., Opt. Phys. 1971, 4, 1597.

(53) Hall, R. W.; Wolynes, P. G. Intermolecular forces and the glass transition. J. Phys. Chem. B 2008, 112, 301-312.

(54) Salsburg, Z. W.; Wood, W. W. Equation of state of classical hard spheres at high density. J. Chem. Phys. 1962, 37, 798-804.

(55) Tool, A. Q. Relation between inelastic deformability and thermal expansion of glass in its annealing range. J. Am. Ceram. Soc. 1946, 29, 240-253.

(56) Narayanaswamy, O. S. A model of structural relaxation in glass. J. Am. Ceram. Soc. 1971, 54, 491-498.

(57) Moynihan, C. T.; Easteal, A. J.; Bolt, M. A.; Tucker, J. J. Am. Ceram. Soc. 1976, 59, 12-16.

(58) Mézard, M.; Parisi, G. A first-principle computation of the thermodynamics of glasses,. J. Chem. Phys. 1999, 111, 1076-1095.

(59) McQuarrie, D. M. Statistical Mechanics; Harper-Collins: New York, 1973.

(60) Stevenson, J. D.; Wolynes, P. G. A universal origin for secondary relaxations in supercooled liquids and structural glasses,. Nat. Phys. 2010, 6, 62-68.

(61) Wisitsorasak, A.; Wolynes, P. G. On the strength of glasses. Proc. Natl. Acad. Sci. U. S. A. 2012, 109, 16068-16072.

(62) Lubchenko, V.; Rabochiy, P. On the mechanism of activated transport in glassy liquids. J. Phys. Chem. B 2014, 118, 13744-13759.

(63) Chan, H. Y.; Lubchenko, V. Pressure in the landau-ginzburg functional: Pascals law, nucleation in fluid mixtures, a meanfield theory of amphiphilic action, and interface wetting in glassy liquids. J. Chem. Phys. 2015, 143, 124502.

(64) Villain, J. Equilibrium critical properties of random field systems: new conjectures. J. Phys. (Paris) 1985, 46, 1843-1852.

(65) Dzero, M.; Schmalian, J.; Wolynes, P. G. Activated events in glasses: The structure of entropic droplets,. Phys. Rev. B: Condens. Matter Mater. Phys. 2005, 72, No. 100201(R), DOI: 10.1103/ PhysRevB.72.100201.

(66) Landau, L. D.; Lifshitz, E. M. Theory of Elasticity; Pergamon Press, 1986 
(67) Landau, L. D.; Lifshitz, E. M. Statistical Mechanics; Pergamon Press: New York, 1980.

(68) Cavagna, A.; Attanasi, A.; Lorenzana, J. Viscoelasticity and metastability limit in supercooled liquids. Phys. Rev. Lett. 2005, 95, 115702.

(69) Rabochiy, P.; Wolynes, P. G.; Lubchenko, V. Microscopically based calculations of the free energy barrier and dynamic length scale in supercooled liquids: The comparative role of configurational entropy and elasticity,. J. Phys. Chem. B 2013, 117, 15204-15219.

(70) Soma, T. Equation of state and bulk modulus under pressure of alkali metals. J. Phys. F: Met. Phys. 1980, 10, 1401.

(71) Mott, P. H.; Roland, C. M. Limits to Poisson's ratio in isotropic materials. Phys. Rev. B: Condens. Matter Mater. Phys. 2009, 80, 132104.

(72) Bevzenko, D.; Lubchenko, V. Stress distribution and the fragility of supercooled melts. J. Phys. Chem. B 2009, 113, 16337-16345.

(73) Bevzenko, D.; Lubchenko, V. Self-consistent elastic continuum theory of degenerate, equilibrium aperiodic solids. J. Chem. Phys. 2014, 141, 174502.

(74) Wyart, M.; Silbert, L. E.; Nagel, S. R.; Witten, T. A. Effects of compression on the vibrational modes of marginally jammed solids. Phys. Rev. E 2005, 72, 051306.

(75) Brito, C.; Wyart, M. Geometric interpretation of previtrification in hard sphere liquids. J. Chem. Phys. 2009, 131, 024504.

(76) DeGiuli, E.; Lerner, E.; Brito, C.; Wyart, M. Force distribution affects vibrational properties in hard-sphere glasses. Proc. Natl. Acad. Sci. U. S. A. 2014, 111, 17054-17059.

(77) Xia, X.; Wolynes, P. G. Microscopic theory of heterogeneity and nonexponential relaxations in supercooled liquids. Phys. Rev. Lett. 2001, 86, 5526-5529.

(78) Bhattacharyya, S. M.; Bagchi, B.; Wolynes, P. G. Facilitation, complexity growth, mode coupling, and activated dynamics in supercooled liquids. Proc. Natl. Acad. Sci. U. S. A. 2008, 105, $16077-16082$.

(79) Nandi, S. K.; Biroli, G.; Tarjus, G. Spinodals with disorder: From avalanches in random magnets to glassy dynamics. Phys. Rev. Lett. 2016, 116, 145701.

(80) Conrad, J. C.; Dhillon, P. P.; Weeks, E. R.; Reichman, D. R.; Weitz, D. A. Contribution of Slow Clusters to the Bulk Elasticity Near the Colloidal Glass Transition. Phys. Rev. Lett. 2006, 97, 265701.

(81) Ikeda, A.; Berthier, L.; Biroli, G. Dynamic criticality at the jamming transition. J. Chem. Phys. 2013, 138, 12 A507.

(82) Billotet, C.; Binder, K. Non-linear relaxation at 1st-order phasetransitions-ginzburg-landau theory including fluctuations. Z. Phys. B: Condens. Matter Quanta 1979, 32, 195-213.

(83) Goldenfeld, N. Lectures on phase transitions and the renormalization group; Addison-Wesley: Reading, MA, 1992.

(84) Ozawa, M.; Berthier, L. Does the configurational entropy of polydisperse particles exist? J. Chem. Phys. 2017, 146, 014502.

(85) Dzero, M.; Schmalian, J.; Wolynes, P. G. Replica theory for fluctuations of the activation barriers in glassy systems. Phys. Rev. B: Condens. Matter Mater. Phys. 2009, 80, 024204.

(86) Casalini, R.; Roland, C. M. Viscosity at the dynamic crossover in $o$-terphenyl and salol under high pressure. Phys. Rev. Lett. 2004, 92, 245702.

(87) Grochala, W.; Hoffmann, R.; Feng, J.; Ashcroft, N. The chemical imagination at work in very tight places. Angew. Chem., Int. Ed. 2007, 46, 3620-3642.

(88) Pan, W.; Vekilov, P. G.; Lubchenko, V. Origin of anomalous mesoscopic phases in protein solutions. J. Phys. Chem. B 2010, 114, $7620-7630$.

(89) Muschol, M.; Rosenberger, F. Liquid-liquid phase separation in supersaturated lysozyme solutions and associated precipitate formation/crystallization. J. Chem. Phys. 1997, 107, 1953-1962. 